\title{
High-resolution millimeter-interferometer observations of the solar chromosphere
}

\author{
S. M. White ${ }^{1}$, M. Loukitcheva ${ }^{2,3}$, and S. K. Solanki ${ }^{3}$ \\ 1 Astronomy Department, University of Maryland, College Park, MD 20742, USA \\ e-mail: white@astro.umd.edu \\ 2 Astronomical Institute, St. Petersburg University, 198504 St. Petersburg, Russia \\ 3 Max-Planck-Institut für Sonnensystemforschung, 37191 Katlenburg-Lindau, Germany \\ Received 10 February 2005 / Accepted 28 April 2006
}

\section{ABSTRACT}

\begin{abstract}
The use of millimeter-interferometer data for the study of chromospheric structure and dynamics is tested using $85 \mathrm{GHz}$ observations with the 10-element Berkeley-Illinois-Maryland Array (BIMA). Interferometer data have the advantage over single-dish data that they allow both high spatial resolution and dense temporal sampling simultaneously. However, snapshot imaging of the quiet solar atmosphere with a small number of dishes is challenging. We demonstrate that techniques are available to carry out this task successfully using maximum entropy deconvolution from a default image constructed from the entire observation: one of our results is that the solar chromosphere at millimeter wavelengths exhibits features that are long-lasting and the map of the entire observation is significant provided that atmospheric phase errors do not prevent deconvolution. We compare observations of quiet Sun, active region and coronal hole targets. The interferometer is not sensitive to the disk emission and the positivity constraint of the maximum entropy algorithm used forces the zero level in the images to be at the temperature of the coolest feature in each field. The brightest features in the images are typically $1000-1500 \mathrm{~K}$ above the zero level, with a snapshot noise level of order $100 \mathrm{~K}$. We use extensive tests to determine whether oscillation power can be recovered from sequences of snapshot images and show that individual sources can be down to quite weak levels at locations in the image where significant flux is present; oscillation power located in cool regions of the image is not well recovered due to the deconvolution method used and may be redistributed to brighter regions of the millimeter image. We then investigate whether the data do show oscillation power using uninterrupted 30-min scans of the target regions. Intensity oscillations with significant power in the frequency range $1.5-8.0 \mathrm{mHz}$ are found in the quiet-Sun and active region targets. For the quiet-Sun region we compare the oscillation properties of network boundaries and cell interiors (internetwork) in the spatially-resolved time series. In agreement with investigations at other wavelengths, in the millimeter data the power in the network tends to be at periods of $5 \mathrm{~min}$ and longer while power in the internetwork is present also at shorter (3-min) periods.
\end{abstract}

Key words. Sun: chromosphere - Sun: radio radiation

\section{Introduction}

Observations of the quiet Sun at millimeter wavelengths are an important technique for studying the solar chromosphere. At these short wavelengths the Sun's atmosphere becomes optically thick in the chromosphere and since the wavelength lies in the Rayleigh-Jeans limit, the measured radio brightness temperature corresponds to the electron temperature in the layer being observed, without the complications of non-equilibrium line formation that have to be taken into account when analyzing visible and UV line observations of the chromosphere. Millimeter-wavelength observations have played an important role in forming thermal models of the chromosphere (e.g., Vernazza et al. 1976, 1981; Fontenla et al. 1990; Gu et al. 1997), and it is natural to ask what they reveal about chromospheric heating (see Solanki 2005).

There have been many single dish observations of the Sun at millimeter and sub-millimeter wavelengths. The limitation of single dish measurements is always spatial resolution: a typical telescope with a diameter of $10 \mathrm{~m}$ has a spatial resolution of $\lambda_{\mathrm{mm}} 30^{\prime \prime}$, where $\lambda_{\mathrm{mm}}$ is the observing wavelength in $\mathrm{mm}$, and thus, e.g., a $10 \mathrm{~m}$ telescope operating at $90 \mathrm{GHz}$ cannot resolve features smaller than about $100^{\prime \prime}$. This problem has been circumvented in two ways: by observing eclipses, when the Moon's limb acts as a knife edge crossing the field of view, and the time dependence of the millimeter flux can be converted into (onedimensional) spatial resolution much finer than the telescope beam (e.g., Tolbert et al. 1964; Hagen et al. 1971; Beckman et al. 1975; Swanson \& Hagen 1975; Shimabukuro et al. 1975; Lindsey et al. 1984; Roellig et al. 1991; Ewell, Jr. et al. 1993); and by going to submillimeter wavelengths (smaller $\lambda$ ), which is possible with the James Clerk Maxwell Telescope (JCMT) and Caltech Submillimeter Telescope (CSO) on Mauna Kea, Hawaii. These telescopes have been able to make images of the solar disk at submillimeter wavelengths with a spatial resolution as small as 20" (Horne et al. 1981; Lindsey \& Jefferies 1991; Bastian et al. 1993a,b; Lindsey et al. 1995; Lindsey \& Kopp 1995). The Nobeyama $45 \mathrm{~m}$ telescope has been able to achieve similar resolution at $\lambda=3 \mathrm{~mm}$ (Kosugi et al. 1986; Irimajiri et al. 1995), while the NRAO 12 m telescope at Kitt Peak can reach a resolution of 40" at $1 \mathrm{~mm}$ (Buhl \& Tlamicha 1970; Kundu 1970, 1971; Lantos \& Kundu 1972).

However, by their nature single-dish observations are not ideal for searching for spatially-resolved time variability in the solar chromosphere. A single dish only samples the flux from a given location when it is pointing there, and thus its duty cycle for sampling the time variation at a given point is poor if the telescope is carrying out imaging since, by definition, most of the time it is pointed at the other locations within the field being mapped (e.g., the raster scan of a $400^{\prime \prime}$ square region by 
Lindsey \& Jefferies 1991, required 45 min with a $1 \mathrm{~s}$ integration at each of 1600 pointings). Single-dish observations are excellent for studying time variability in the total flux from a fixed location (i.e., the spatially-integrated flux within the telescope beam; e.g., Simon \& Shimabukuro 1971; Kundu \& Schmahl 1980; Lindsey \& Roellig 1987; Kurths et al. 1988; Kopp et al. 1992), but cannot simultaneously image. Multibeam receivers on single-dish telescopes can speed up this process but do not change the nature of the data. The attraction of interferometer observations is that they offer the opportunity for simultaneously achieving higher temporal and spatial resolution observations than can be achieved with a single dish telescope. Since for a single dish telescope the field of view is the same as the spatial resolution, the telescope must have a large aperture and be physically driven to different pointing locations in order to map a region. By contrast, an interferometer with smaller dishes (sensitivity not being much of an issue for the Sun) can make an image with many resolution elements across the field of view, and achieves high time resolution because it forms an image without repointing and thus can stare at the same field of view continuously, providing complete sampling of the light curve in each resolution element. On the other hand, an interferometer has two disadvantages: it is insensitive to the background flux of the Sun, which is resolved out because it comes from a source too large to be sampled by the interferometer spacings; and an interferometer is equivalent to a sparsely filled aperture, meaning that it does not completely sample the spatial structure within the field of view at any instant, and this creates difficulties in reconstructing images (discussed further below). Interferometer observations of the (non-flaring) Sun at millimeter wavelengths have been carried out since the 1970s (e.g., Labrum et al. 1978; Labrum 1978; Janssen et al. 1979; Belkora et al. 1992; White \& Kundu 1994), but generally not with a sufficient number of telescopes for snapshot imaging to be feasible. Here we discuss interferometer images of the Sun made at a wavelength of $3.5 \mathrm{~mm}$ with the Berkeley-Illinois-Maryland Array (BIMA), which, with the largest number of dishes of any existing millimeter interferometer, is the telescope best suited for such observations.

It is not at all clear, a priori, that millimeter interferometer observations of the quiet Sun will be successful due to the dynamic nature of the chromosphere. Evidence from all wavelengths is that the chromosphere is a turbulent layer, constantly pushed from below by convective motions and waves, and being bombarded from above by energetic particles. Radiative energy loss timescales are quite short, and motions are common: Zirin (1996) comments that "In high-resolution movies the $\mathrm{H} \alpha$ chromosphere is a seething, oscillating mass of fibrils". At centimeter wavelengths radio images of the Sun are usually dominated by very bright, relatively compact sources in the corona above active regions, but at millimeter wavelengths the image consists of temperature fluctuations on the optically thick layer in the chromosphere corresponding to the frequency observed, and these will fill the field of view with low-contrast features. Such an image requires many components to describe it adequately and, as we discuss below, a single instantaneous observation, even with BIMA, does not contain enough information to reconstruct the brightness distribution within the field of view. A standard technique used by interferometers to overcome the instantaneously sparse sampling of spatial scales is to integrate over a long period, using the Earth's rotation and the changing elevation of the source to fill in the sampling (the technique of "Earth-rotation synthesis"), but this can only work if the target observed is steady over the time period used: if not, then samples at different times refer to different brightness distributions and cannot be combined. If the chromosphere's millimeter emission is such that its pattern changes from minute to minute, then clearly Earth-rotation synthesis will not work. Single dish observations do not suffer from this problem, since each point is only observed briefly, but equally single-dish observations cannot easily be used to investigate this issue.

In this paper we analyze millimeter-interferometer observations of the solar chromosphere obtained with BIMA and look for spatially-resolved oscillation power in the observations. We observe and compare an active region, quiet-Sun region and a coronal hole. The imaging technique used for the millimeter data is discussed in some detail. An important question is the sensitivity of the observations to oscillation power, and we present extensive tests of this issue. In the companion paper by Loukitcheva et al. (2006) we compare the observed millimeter intensity variations derived in the present analysis with the oscillation properties predicted by the Carlsson \& Stein models (Carlsson \& Stein 1995, 1997) and discuss the reasons for differences that we find.

\section{Radio emission from the quiet Sun at millimeter wavelengths}

Radio emission at millimeter wavelengths becomes optically thick in the solar chromosphere due to the opacity contributed by thermal bremsstrahlung. At temperatures below $10^{4} \mathrm{~K}$ the dominant species ( $\mathrm{H}$ and $\mathrm{He}$ ) are mostly neutral, but the total electron and proton densities can remain high because the total density increases rapidly with depth and compensates for the decrease in fractional ionization. Below $5000 \mathrm{~K}$ (i.e., in the temperature minimum) essentially all hydrogen is neutral. Free electrons are still present at these temperatures due to ionization of metals such as sodium, but there are insufficient free protons to provide significant free-free opacity. In this situation a mechanism involving free electrons and neutral $\mathrm{H}$ dominates the radio opacity: an electron polarizes an $\mathrm{H}$ atom and the interaction between the electron and the dipolar atom provides opacity known as " $\mathrm{H}^{-}$opacity". However, at the wavelength we investigate here, $3.5 \mathrm{~mm}(85 \mathrm{GHz})$, we can assume that electron-proton bremsstrahlung still dominates the radio opacity.

The expression for this opacity from the top of the solar corona down to a height $h_{0}$ in the chromosphere is given by

$\tau=\frac{.01}{f^{2}} \int_{h_{0}}^{\infty} \frac{n_{\mathrm{e}}\left(n_{p}+4 n_{\mathrm{He}^{++}}\right)(17.6+1.5 \ln T-\ln f)}{T^{1.5}} \mathrm{~d} h$

where $f$ is the frequency, $n_{\mathrm{e}}$ the electron number density, $n_{p}$ the proton number density, $n_{\mathrm{He}^{++}}$the fully-ionized Helium number density and $T$ is the temperature. Apart from $f$, all these quantities depend on height and this expression can only be evaluated in the context of a model atmosphere in which both the density and the ionization fraction are specified as a function of height. Radio measurements of $T$ (which is the same as the radio brightness temperature since the radio measurement is in the Rayleigh-Jeans limit and the source is optically thick) as a function of $f$ provide constraints for such models (e.g., Vernazza et al. 1976, 1981; Fontenla et al. 1990; Ewell, Jr. et al. 1993; Gu et al. 1997). At millimeter wavelengths the temperature varies much more slowly than electron density as a function of height. As a result, the optically thick frequency, i.e. the frequency at which optical depth unity $(\tau=1)$ is reached at a given height $h_{0}$, depends predominantly on the density gradient:

$f^{2} \propto \int_{h_{0}}^{\infty} n_{\mathrm{e}} n_{p} \mathrm{~d} h$ 
With only a weak dependence on the temperature gradient, this result implies that the optically thick surface at a given frequency is determined by the column density above the surface and is relatively independent of the temperature on the surface. Thus, heating in the chromosphere in the layers around $\tau=1$ should lead directly to an increase in the millimeter brightness temperature since the integrated column above the heated location will not change greatly. Heating in the optically thin layers above the $\tau=1$ surface, on the other hand, decreases the optically thin contribution from the locally heated material but can lead to an increase in the column density of $n_{\mathrm{e}}$ as heat propagates downwards to the denser layers and causes them to expand, pushing the $\tau=1$ layer up the temperature gradient. This is likely to occur on a timescale slower than that of the heating, but may well affect the radio brightness temperature and thus also result in sensitivity of mm-wavelength radiation to heating that is localized in a layer away from the $\tau=1$ surface (Loukitcheva et al. 2004).

\section{BIMA observing procedure and data analysis}

\subsection{BIMA observations}

BIMA is a 10-element interferometric array consisting of $6.1 \mathrm{~m}$ diameter dishes (Welch et al. 1996). The BIMA observations followed the standard practice of alternating observations of an unresolved strong point source for phase calibration with observations of the target regions. In this case, we alternated between the three targets (active region, quiet Sun and coronal hole) with scans 31 min long (29 min of integration) interspersed with scans of the calibration source $1058+015$. The three target regions are shown on an EIT $195 \AA$ Fe XII image in Fig. 1. BIMA tracks a fixed point on the solar surface by correcting for solar rotation in real time. The correlator was set to observe two $800 \mathrm{MHz}$-wide sidebands at 85 and $88 \mathrm{GHz}$. BIMA was in its most compact ("D") configuration for these observations, resulting in a nominal resolution of order $10^{\prime \prime}$, but minimizing the effects of atmospheric phase distortions on the data. Phase variations in the data due to diurnal changes in the lengths of the optical fibers connecting the antennas to the central building amounted to several thousand degrees, but these are measured accurately by the online system and removed during the calibration process. Errors due to atmospheric phase fluctuations appeared to be relatively small until the end of the observation when the Sun was low on the western horizon, and we exclude the last (fifth) scan of the active region for this reason, leaving four scans of each of the three target regions. The time resolution was $4 \mathrm{~s}$ with an integration time of $3 \mathrm{~s}$.

Solar observations with BIMA do not use the standard amplitude calibration method because the Sun fills the antenna beam with a brightness temperature of order $7000 \mathrm{~K}$, and requires the receivers to operate in a regime quite different from normal observations where the system temperature is of order $300 \mathrm{~K}$. In order to measure the system gain at the Sun's brightness temperature, a modified version of the conventional chopper wheel method (Ulich \& Haas 1976) has been implemented by Plambeck and Bock (described in Plambeck 2000). Each chopper wheel on the BIMA antennas carries in one quadrant a partially transparent vane (a "pellicle") with a transmission factor of order $70 \%$ (measured in the laboratory prior to installation). The pellicles can be rotated into the beam path and the receiver response at $70 \%$ of the Sun's brightness temperature can then be compared with the response when the Sun is observed directly. A suitable modification of the Ulich \& Haas (1976) formula allows

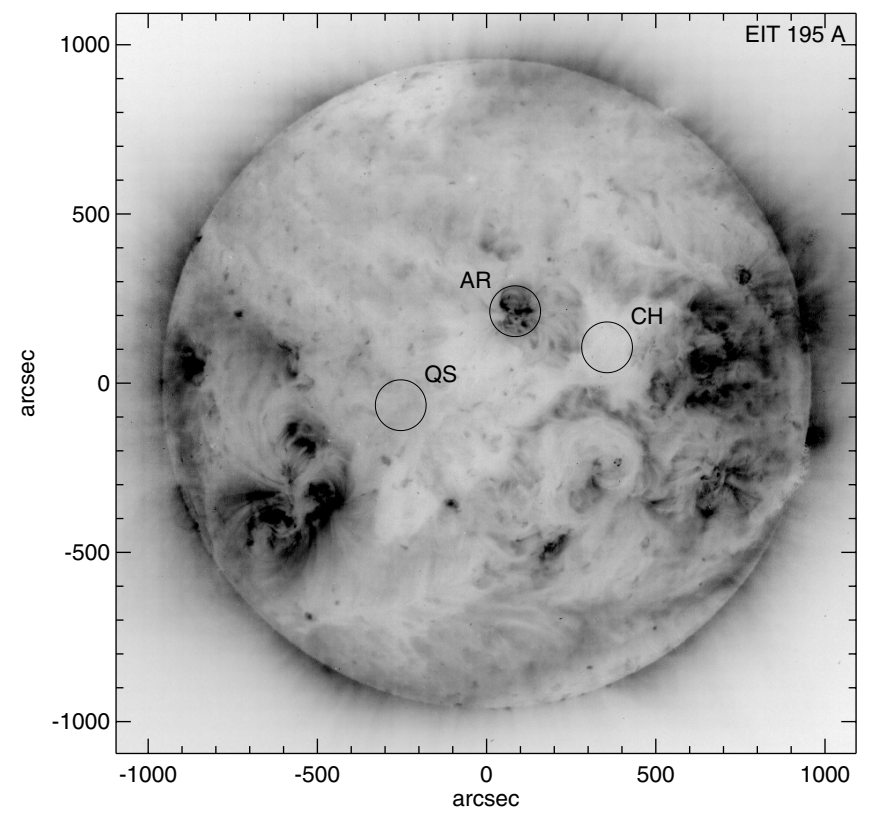

Fig. 1. An EIT $195 \AA$ A image of the Sun at 19:12 UT on 2003 August 31 (reversed color table, so that dark features represent bright emission) together with circles showing the targets observed by BIMA. The circles have a diameter of $150^{\prime \prime}$, just slightly larger than the half-power beam width of the BIMA antennas at $85 \mathrm{GHz}$, and are labelled for the appropriate feature: "AR" (active region), "QS" (quiet Sun), and "CH" (coronal hole).

the gain to be computed, under the assumption that the Sun's temperature at $3 \mathrm{~mm}$ wavelength is $7000 \mathrm{~K}$, which should be appropriate (e.g., see the survey by Urpo et al. 1987). This correction is implemented by a customized module in the on-line correlator software. The dielectric beamsplitter used for the vane is polarization-dependent and this introduces some uncertainty in the amplitude calibration. We cross-checked the amplitude calibration scheme by comparing BIMA data with three other sources: Nobeyama Radio Polarimeter data for a flare at $80 \mathrm{GHz}$ that BIMA observed at $85 \mathrm{GHz}$; estimates of thermal free-free emission during the decay phase of flares based on GOES soft X-ray data; and total power measurements on BIMA antenna 3, which has an older Schottky receiver that is more linear than the receivers on the newer BIMA antennas. From this comparison, we presently estimate a conservative uncertainty in the absolute interferometer fluxes of $30 \%$.

The total power level on antenna 3 at BIMA is known to show oscillations associated with the water chiller on the antenna that can propagate into the visibility data. The oscillation was seen in total power with periods ranging from 13 to 16 min during this observation but there was no obvious sign of such oscillations in the visibility data; in any case, at those long periods they should not interfere with the search for periods from 3 to $5 \mathrm{~min}$. None of the other antennas show such oscillations.

\subsection{Image processing}

The advantage of using an interferometer to observe the chromosphere is that it can achieve better spatial resolution than a single dish observation at the same frequency. The disadvantage is that the interferometer is insensitive to flux on spatial scales larger than the angular scale corresponding to the Fourier fringe spacing of the shortest antenna spacing, and in particular it is insensitive to the background solar disk level. We know that 
millimeter images of the solar atmosphere should consist of features with contrast of order hundreds to $1-2$ thousand $\mathrm{K}$ against a flat background of order $7000 \mathrm{~K}$, and relative to that background the images will have patterns of positive and negative emission. This is seen in, e.g., high-resolution interferometer images of the solar chromosphere at microwave frequencies (4-22 GHz: Erskine \& Kundu 1982; Gary et al. 1990; Bastian et al. 1996; Benz et al. 1997).

Since the images are expected to have emission in many pixels but with relatively low contrast, the appropriate deconvolution method is maximum entropy ("MEM") rather than the "CLEAN" process usually used for high-contrast images with small bright features (Cornwell 1988). We attempted to use the "CLEAN" procedure but it failed to produce maps that were believable, presumably because of the combination of high sidelobes (up to $50 \%$ in snapshot maps) and the presence of flux in just about every pixel in the image. Even small amounts of flux being redistributed by the point-source response causes problems in a low-contrast image because at any location you can get spurious contributions from pixels all across the image, whose deconvolution by "CLEAN" would require excessively large numbers of "CLEAN" components and a very slow "CLEAN" step. We used the maxen program in the MIRIAD package for MEM deconvolution ${ }^{1}$, allowing the program to find the total flux, and restored the deconvolved images with restoring beams of $10^{\prime \prime}$ for the images presented here. Since maxen has a positivity constraint, the deconvolution process sets the zero level at the flux of the most negative real feature in the image and forces all other features to be brighter than this. Therefore the zero level in the images has little meaning. The total power measurements from the receivers indicated that the active region target showed a total power about $2 \%$ larger than the quiet Sun region and about $1 \%$ larger than the coronal hole.

The $6.1 \mathrm{~m}$ BIMA antennas have a half-power beam width of $137^{\prime \prime}$ at $85 \mathrm{GHz}$ and the first null is at a radius of $170^{\prime \prime}$ from the center of the primary beam (Lugten 1995). We made images 128 pixels square with a cell size of $3^{\prime \prime}$, and looked for flux in the inner region of the image, i.e., a circular region of diameter 192", which extends beyond the half-power size. However, generally flux in the deconvolved images is confined to within the halfpower region.

\subsection{Snapshot imaging and BIMA sensitivity}

One of the goals of this investigation is to look for oscillations in the chromospheric emission at timescales from 3 to $5 \mathrm{~min}$, requiring imaging to be carried out on much shorter timescales, i.e., snapshot imaging. We made images at intervals of $15 \mathrm{~s}$ throughout the day, with each interval including about 4 integrations. Maximum entropy deconvolution of these snapshots without using a priori models did not produce satisfactory images. The reasons for this can be understood as follows. When all ten antennas were operating (only true for a period after $21 \mathrm{UT}$ ), BIMA obtained 45 complex numbers in a snapshot (one for each antenna pairing), so that at best an image with about 30 distinct emission components in it can be reconstructed (a single point

\footnotetext{
1 We compared the results from maxen with the MEM deconvolution programs in the AIPS package maintained by the National Radio Astronomical Observatory, VTESS (which uses the Cornwell entropy measure rather than the Gull entropy measure used by maxen, but also has a positivity constraint), and UTESS (which has no positivity constraint). VTESS gave essentially the same results as maxen, with the total fluxes determined by the two programs agreeing to within $10 \%$, but UTESS failed to converge to a suitable solution.
}

source requires 3 pieces of information: a flux and two position coordinates). The BIMA field of view, however, contains about 400 resolution elements (140" field with $10^{\prime \prime}$ beam, requiring 4 samples per beam), and we expect flux to be present everywhere, unlike the case at longer wavelengths or for flares where a few bright sources dominate. Therefore a snapshot does not contain enough information to reconstruct the brightness distribution completely.

We address this problem by using a priori models as default images for each snapshot. The models used are the results of deconvolving the data for each target region for the whole day. Earth rotation synthesis then gives us enough information to reconstruct the brightness distribution, provided that it is reasonably stable over the day. At a gross level this was found to be the case, particularly for the active region target. Using a model as a default image for deconvolution is the well-discussed process of supplying a priori information (Cornwell \& Braun 1989). The deconvolution procedure should then only modify the model where the snapshot data require it, i.e., only where the data contain features that are not consistent with the default model to within the noise level. In a sense, we are asking the deconvolution process to reconstruct changes in a small number of pixels where the emission changes by amounts that are significantly larger than the noise level (smaller changes are irrelevant), rather than in every pixel in the image that contains flux. This approach should minimize the effects of the snapshot $u, v$ distribution varying throughout the day, but it complicates the issue of determining the sensitivity of the method to fluctuating sources. This approach produced snapshot maps of excellent quality that clearly showed changes with time, whereas images constructed without using a priori information were poor (with both CLEAN and maximum entropy deconvolution).

The tests carried out to determine the believable level of fluctuations in the images were twofold. Firstly, images in the two sidebands at 85 and $88 \mathrm{GHz}$ should be essentially identical since the corresponding optically thick layers in the chromosphere are so close to one another. The snapshot images at the two frequencies were made independently using identical methods and compared. The two images were always consistent with one another. The noise level in the snapshot difference maps was typically of order 0.006 solar flux units $(\mathrm{sfu})$ per beam $\left(1 \mathrm{sfu}=10^{4} \mathrm{Jy}\right)$ or $100 \mathrm{~K}$ brightness temperature (for a $10^{\prime \prime}$ beam).

Secondly, we added model point sources of different fluxes to snapshot visibilities and mapped the resulting data as before. This test indicated that model sources as weak as $0.001 \mathrm{sfu}$ $(17 \mathrm{~K})$ could be recovered adequately in regions of the images where the flux is high, but in other regions (regions with low flux) sources as strong as $.05 \mathrm{sfu}(800 \mathrm{~K})$ could not be recovered. We present an explanation for this behaviour later in discussing tests of the recovery of oscillating sources.

\section{Comparison of millimeter images with other diagnostics}

In order to provide a context for understanding the millimeter observations, we compare them with the following data: (i) a longitudinal magnetogram obtained at 19:11 UT by the Michelson Doppler Interferometer (MDI) on the Solar and Heliospheric Observatory (SOHO); (ii) a $195 \AA$ image (dominated by an Fe XII line formed in the corona at $1.5 \times 10^{6} \mathrm{~K}$ ) at 19:13 UT from the Extreme-ultraviolet Imaging Telescope (EIT) on SOHO; (iii) a $304 \AA$ EIT image at 19:19 UT dominated by the $\mathrm{He}$ I line formed at about $10^{5} \mathrm{~K}$; (iv) a line-center $\mathrm{H} \alpha$ 


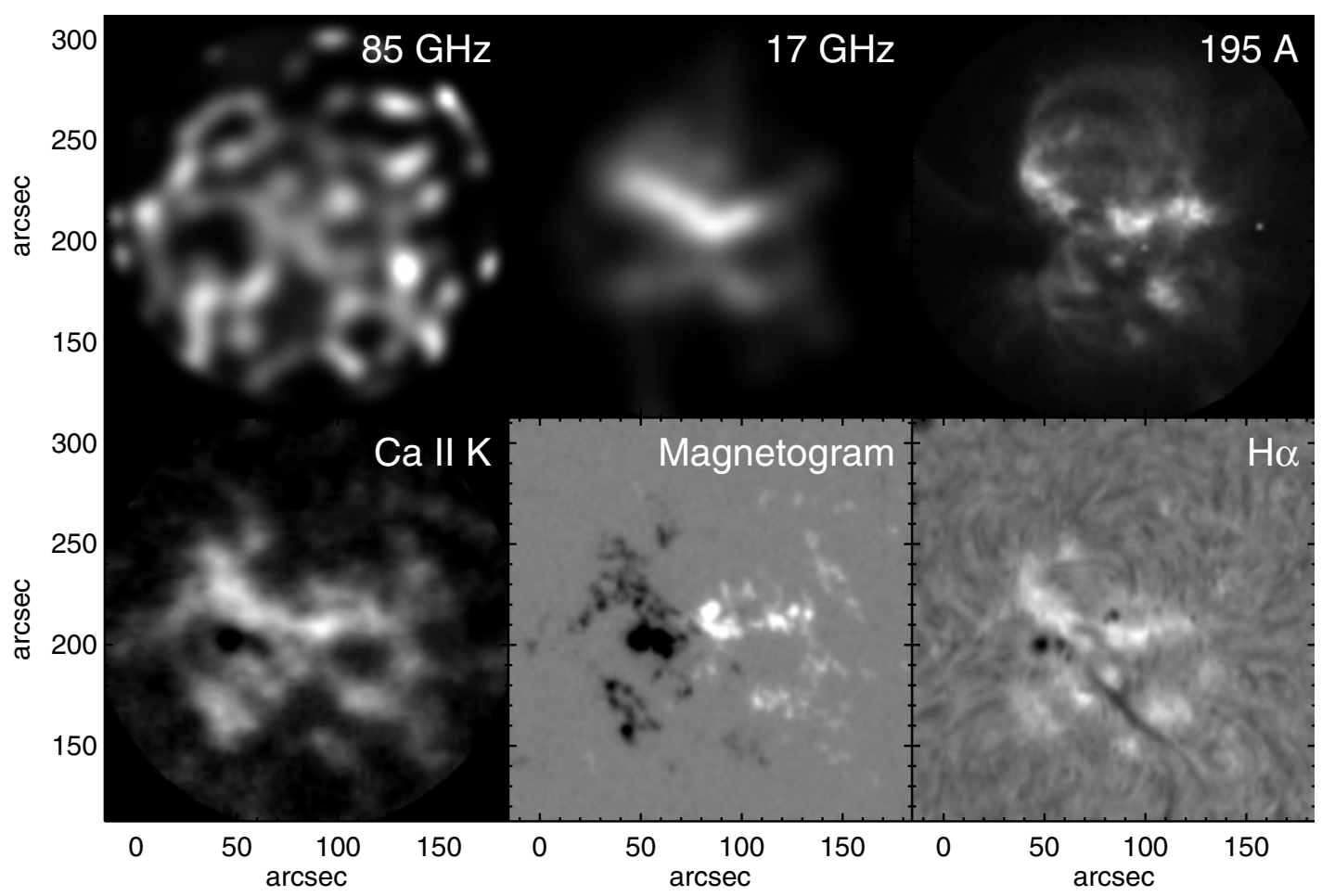

Fig. 2. Comparison of the active region (AR) field of view at a number of wavelengths (labelled): the BIMA image at $85 \mathrm{GHz}$, the NoRH image at $17 \mathrm{GHz}$, the EIT $195 \AA$ A Fe XII image, the BBSO Ca II K image, the MDI longitudinal magnetogram and the BBSO H $\alpha$ image. The 85 GHz image is of the data from the first two 30-min scans and has been corrrected for the primary beam response and truncated in a $96^{\prime \prime}$ radius field of view, which is also imposed on the $17 \mathrm{GHz}, 195 \AA$ and $\mathrm{Ca}$ II K image. The magnetogram display range is $\pm 500 \mathrm{G}$, at $17 \mathrm{GHz}$ the display range is 1.0 to $2.5 \times 10^{4} \mathrm{~K}$, and at $85 \mathrm{GHz}$ the display range is 0 to $2000 \mathrm{~K}$ (above the background disk level of order $7000 \mathrm{~K}$ ).

image obtained by the Big Bear Solar Observatory (BBSO) at 19:02 UT; (v) a BBSO Ca II K line image acquired at 16:37 UT; and (vi) a $17 \mathrm{GHz}$ image from the Nobeyama Radio Heliograph (NoRH) made by averaging 60 samples 1 min apart covering the time range 23:00-23:59 UT (after sunrise in Japan) with solar rotation removed from the brighter features. Since it is close to the mid-time of the BIMA observations, we use 19:00 UT as the reference time and all images were rotated to the common time for comparison. The $85 \mathrm{GHz}$ images used here have a spatial resolution of $10^{\prime \prime}$. The $17 \mathrm{GHz}$ image has a spatial resolution of $12^{\prime \prime}$, while all the optical and EUV images have resolutions of order 2-4". The line-center $\mathrm{H} \alpha$ and $\mathrm{Ca}$ II $\mathrm{K}$ emissions are believed to form in the chromosphere at temperatures of order $6000-8000 \mathrm{~K}$. At $17 \mathrm{GHz}$ the solar disk has a brightness temperature of $10000 \mathrm{~K}$, so this frequency is also a chromospheric diagnostic in principle and is optically thick at a height above the formation levels of $\mathrm{H} \alpha$ and $\mathrm{Ca}$ II K. However, in active regions coronal material can also contribute at $17 \mathrm{GHz}$ due to optically thin bremsstrahlung from the hot plasma in coronal loops. Since optically thin bremsstrahlung has a brightness temperature spectrum $\propto f^{-2}$, where $f$ is frequency, the brightness temperature contribution of coronal material at $85 \mathrm{GHz}$ should be just $4 \%\left(\approx(85 / 17)^{-2}\right)$ of its contribution at $17 \mathrm{GHz}$. Coalignment of target region images at different wavelengths was based on full-disk images; fine coalignment was possible using the active region field in which the sunspot is clearly visible at many different wavelengths.

\subsection{Active region}

The small active region NOAA 10448 north of the solar equator (Fig. 1) was the target for these observations. Images of the region at six wavelengths are shown in Fig. 2. The largest sunspot in the region is actually at the trailing (eastern) end of the region. Clear depressions over this sunspot are seen in all six panels: at $85 \mathrm{GHz}$, this is the location of the weakest emission (the zero level in the maximum entropy maps). The loop of bright emission encircling this sunspot in the $\mathrm{Ca}$ II $\mathrm{K}$ image is essentially reproduced in the $85 \mathrm{GHz}$ image. The $\mathrm{H} \alpha$ image shows a narrow filament in the south of the region that is not a prominent feature at any of the other wavelengths.

The brightest emission in the $17 \mathrm{GHz}, 195 \AA \mathrm{Ca}$ II K and $\mathrm{H} \alpha$ images consists of a broad upright "V" of emission north of the center of the region. This feature is not conspicuously present in the $85 \mathrm{GHz}$ image, although there is certainly millimeter emission in its vicinity. The nature of this feature (coronal or chromospheric) is not obvious from the images: the $195 \AA$ image suggests that it consists of discrete features low in the atmosphere, while its presence in the $\mathrm{Ca}$ II $\mathrm{K}$ and $\mathrm{H} \alpha$ images clearly indicates a chromospheric component. In the $17 \mathrm{GHz}$ image this feature has a peak brightness temperature contribution of order $15000 \mathrm{~K}$ (median of order $10000 \mathrm{~K}$ ), and if it is optically thin bremsstrahlung from coronal material then it should contribute a peak of $600 \mathrm{~K}$ (median $400 \mathrm{~K}$ ) to the $85 \mathrm{GHz}$ image. The actual brightness temperatures in the $85 \mathrm{GHz}$ image in this region are in the range 900 (median) to $1500 \mathrm{~K}$ above the sunspot level, so the optically thin coronal contribution from this feature may be partially masked by a larger optically thick chromospheric contributions along the line of sight at $85 \mathrm{GHz}$.

Comparisons of the $85 \mathrm{GHz}$ and magnetic field data with the Ca II K image of the region are shown in more detail in Fig. 3. In this case the $85 \mathrm{GHz}$ data and the magnetic field measurements (represented by the $\pm 40 \mathrm{G}$ contours plotted over the Ca II K image) are from times closer to the acquisition time of the Ca II K 


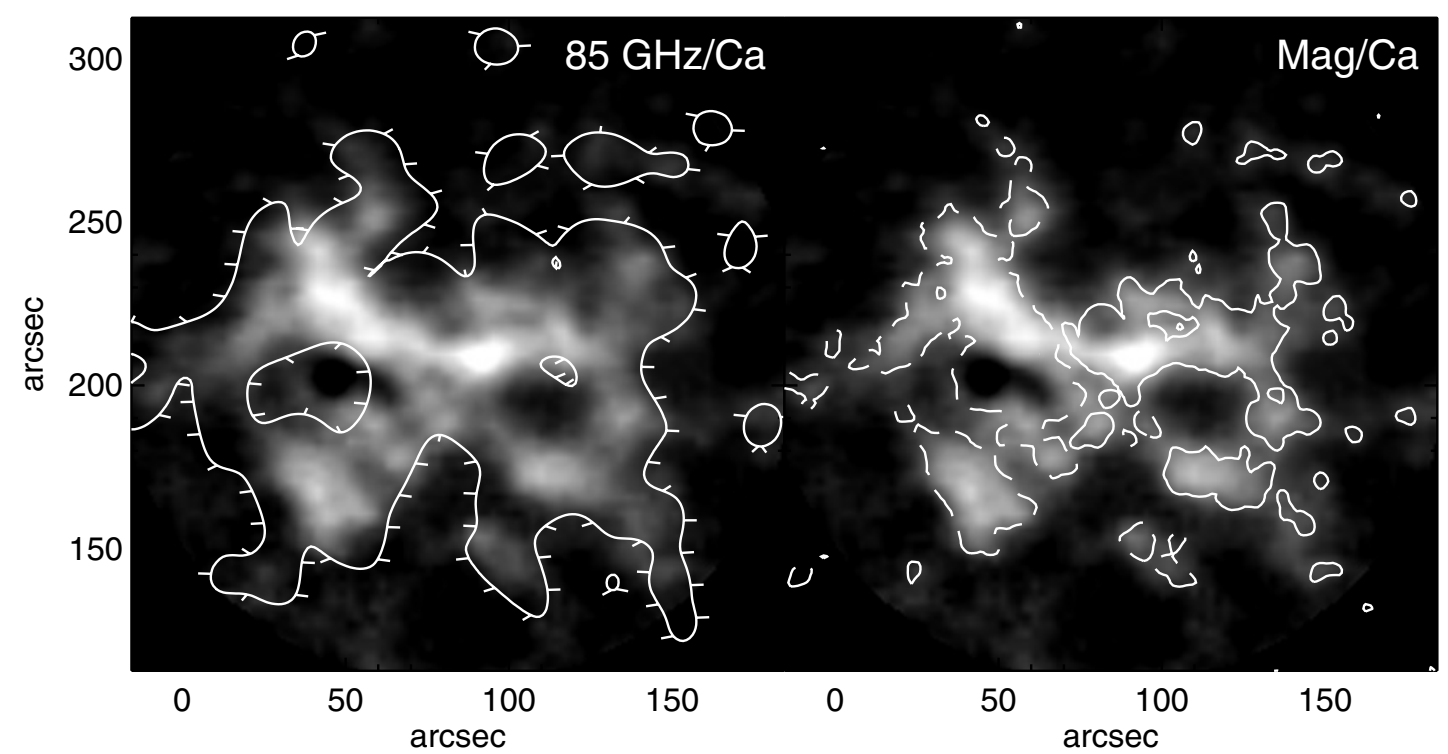

Fig. 3. Overlays of contours of the BIMA $85 \mathrm{GHz}$ emission (left) and the MDI longitudinal magnetic field (right) on the BBSO Ca II K 16:37 UT image (greyscale) for the active region field of view. The millimeter contour is for the second active region scan at a brightness temperature of $500 \mathrm{~K}$, while the magnetic field contour is at $\pm 40 \mathrm{G}$.

image (16:37 UT). In the left panel we plot a single $85 \mathrm{GHz}$ contour at a brightness temperature of $500 \mathrm{~K}$ (about $20 \%$ of the peak). This contour follows the outer edge of the bright $\mathrm{Ca}$ II $\mathrm{K}$ emission extremely well, and confirms the success of the $85 \mathrm{GHz}$ imaging. Note that there are two "holes" interior to the bright $\mathrm{Ca}$ II K emission of different character: the hole on the left lies over the sunspot and is prominent in the $85 \mathrm{GHz}$ image, while the hole to the right of center lies over weak magnetic fields and is simply a low-activity region: it is not as prominent in the $85 \mathrm{GHz}$ image. The high degree of association between strong magnetic fields and bright $\mathrm{Ca}$ II $\mathrm{K}$ emission is obvious in the right hand panel, with the obvious exception of the sunspots. The visual impression of a high degree of correlation of the $85 \mathrm{GHz}$ emission with the $\mathrm{Ca}$ II $\mathrm{K}$ emission is confirmed quantitatively. Within a radius of $80^{\prime \prime}$ the linear correlation coefficient (Pearson's) between the two images is 0.60 , and the rank correlation coefficient (Spearman) is 0.70 , while for the magnetic field strength the correlations with $\mathrm{Ca}$ II $\mathrm{K}$ are 0.44 and 0.70 , respectively. We take this excellent correlation as confirmation of the success of the BIMA observations. Also note that these correlation coefficients cannot take account of temporal variations: the $85 \mathrm{GHz}$ images are averages over an $8 \mathrm{~h}$ period, while the images at most of the other wavelengths are instantaneous snapshots.

The total flux recovered in the BIMA image is about $5 \mathrm{sfu}$; for comparison, the BIMA primary beam filled with a source at $7000 \mathrm{~K}$ should produce $77 \mathrm{sfu}$ at $85 \mathrm{GHz}$. The peak flux is around .09 sfu per beam, corresponding to a brightness temperature of order $1600 \mathrm{~K}$ (without primary beam correction). Recall that this peak brightness temperature really reflects the range in temperature from the coolest (the sunspot) to the hottest feature in the image. This range is similar to that seen in probably the highest quality single-dish millimeter image, that of Bastian et al. (1993a) with a resolution of $21^{\prime \prime}$ at $0.85 \mathrm{~mm}$. They observed an active region in which a sunspot appeared as a depression with a temperature of $6000 \mathrm{~K}$, while the surrounding active region was up to $1000 \mathrm{~K}$ brighter. However, in standard chromospheric models such as Vernazza et al. (1981) and Fontenla et al. (1990, 1991), we expect that the $3.5 \mathrm{~mm}$ emission comes from somewhat higher in the atmosphere than the $0.85 \mathrm{~mm}$ emission, and thus larger variations in brightness temperature are possible. The higher spatial resolution of our observations are another likely cause of the higher brightness temperature contrasts. Lindsey et al. (1990) also observed sunspots at $0.85 \mathrm{~mm}$ and did not find them to be significantly darker than the quiet Sun level (although clearly darker than the surrounding plage in active regions), but Lindsey \& Kopp (1995) carried out a more extensive analysis and concluded that sunspot umbrae at $0.85 \mathrm{~mm}$ can be considerably cooler than the quiet Sun, as low as $4000 \mathrm{~K}$ (at least $2000 \mathrm{~K}$ cooler than the chromosphere at that wavelength). The sunspot in Fig. 2 is not large enough for us to treat the penumbra and the umbra separately here.

\subsection{Quiet Sun}

The target for this observation was a region of diffuse coronal emission in the EIT $195 \AA$ image that clearly lay neither over an active region nor in a coronal hole (Fig. 1). Images of the region at six wavelengths are shown in Fig. 4. This field of view is distinguished by the almost complete absence of strong magnetic fields in the magnetogram: the largest field strength is $120 \mathrm{G}$, but only $5 \%$ of the pixels shown in Fig. 4 have field strengths in excess of $20 \mathrm{G}$. The $\mathrm{H} \alpha$ image is correspondingly devoid of prominent features. The BIMA image at $85 \mathrm{GHz}$ shows a pattern of bright and dark features resembling the cell pattern of the network, while the $17 \mathrm{GHz}$ image is dominated by a depression on the east side of the field of view that is $2000 \mathrm{~K}$ cooler than the surrounding $10000 \mathrm{~K}$ disk. By contrast, the brightest feature at $17 \mathrm{GHz}$ is only $1000 \mathrm{~K}$ above the disk level. The total flux in the $85 \mathrm{GHz}$ image is about $4 \mathrm{sfu}$.

The prominent $17 \mathrm{GHz}$ depression is not evident as a feature at any other wavelength apart from $85 \mathrm{GHz}$, where it is also the coolest location in the image, $400 \mathrm{~K}$ cooler than the median level in the image but $2000 \mathrm{~K}$ cooler than the brightest feature. The location of the depression at $85 \mathrm{GHz}$ is not exactly the same as in the $17 \mathrm{GHz}$ image, but it is a steady feature during the observations and since the $17 \mathrm{GHz}$ image corresponds to a period at the end of the BIMA observation, we are confident that they are the same feature. Inspection of MDI data for several days 


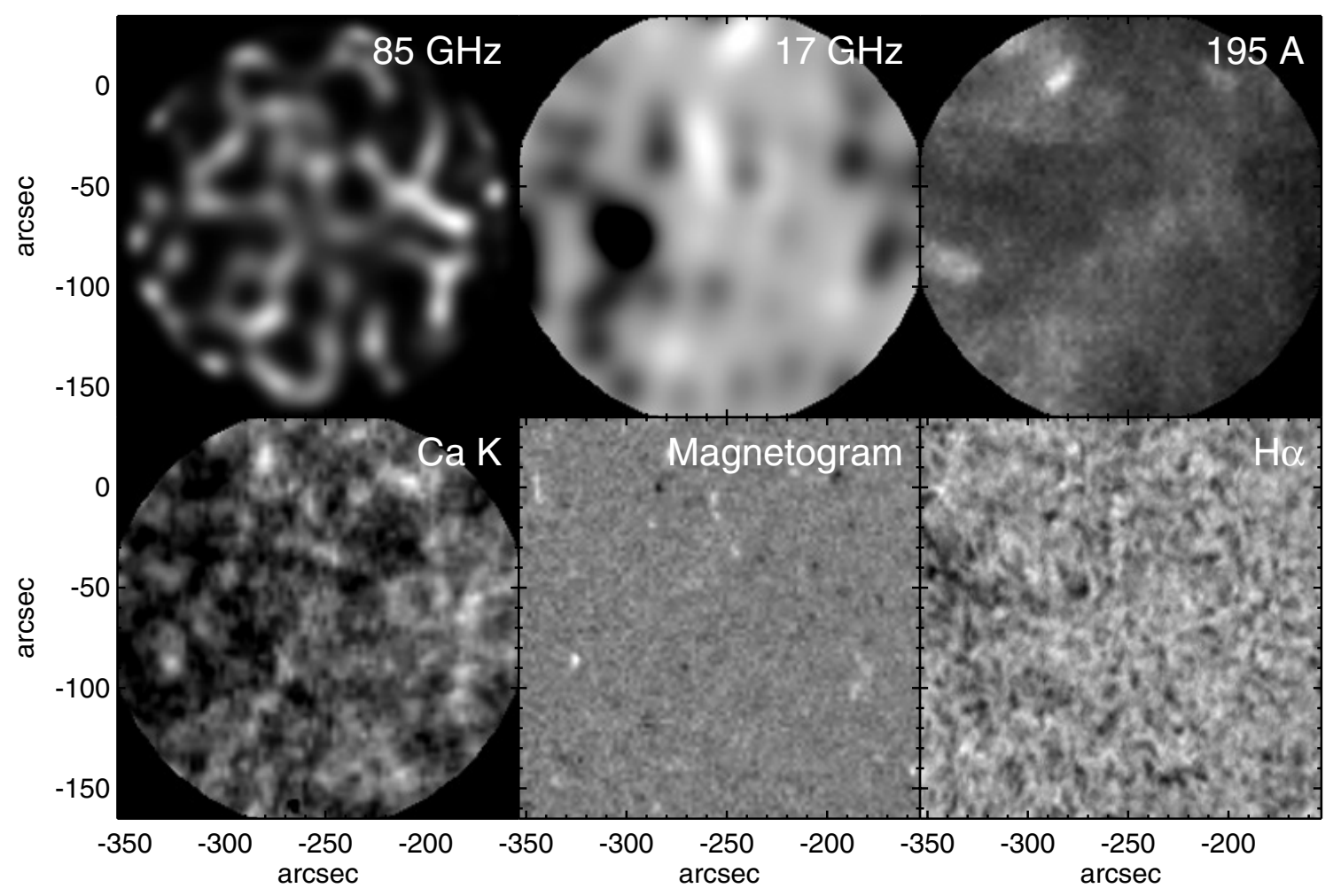

Fig. 4. Comparison of the quiet Sun (QS) field of view at a number of wavelengths in the same format as Fig. 2. The magnetogram display range is $\pm 100 \mathrm{G}$, at $17 \mathrm{GHz}$ the display range is 9000 to $10900 \mathrm{~K}$, and at $85 \mathrm{GHz}$ the display range is 0 to $2000 \mathrm{~K}$ (with correction for primary beam response).

before and after the day of the BIMA observation did not show any optical feature at this location. The $17 \mathrm{GHz}$ and $85 \mathrm{GHz}$ images have a linear (Pearson's) correlation coefficient of 0.23 , and a rank (Spearman) correlation coefficient of 0.19 , but due to the absence of strongly magnetic features in this field of view, the degrees of correlation between the other images at different wavelengths are generally low. While this may raise questions about features in the $85 \mathrm{GHz}$ images, we note that the quiet-Sun and coronal hole scans were interleaved with the active region scans during the observation and the data from all three targets were processed in exactly the same way: the excellent correlation of the $85 \mathrm{GHz}$ active-region data with other wavelengths (Fig. 3) establishes that the features in the other $85 \mathrm{GHz}$ target regions should be valid.

Gary et al. (1990) measured brightness temperature contributions of up to $15000 \mathrm{~K}$ from the network at a wavelength of $3 \mathrm{~cm}$. Bastian et al. (1996) used the VLA to observe the quiet Sun at wavelengths of 2 and $1.3 \mathrm{~cm}$. They determined the absolute level of emission using total power measurements from the VLA antennas themselves, accurate to about $20 \%$. They found a temperature range of about $2000 \mathrm{~K}$ across their field of view at $1.3 \mathrm{~cm}$ and $3000 \mathrm{~K}$ at $2 \mathrm{~cm}$.

\subsection{Coronal hole}

The coronal hole target chosen is just to the west of disk center (Fig. 1). Images at six wavelengths are shown in Fig. 5. In contrast to the quiet Sun region, this coronal hole field contains a number of strong magnetic features, almost all of negative polarity. $12 \%$ of the pixels in this field have field strengths exceeding $20 \mathrm{G}$ and $5 \%$ exceed $50 \mathrm{G}$, with a maximum field strength of $300 \mathrm{G}$. However, as the $195 \AA$ image shows, the corona has very low density over the coronal hole. Again the $\mathrm{H} \alpha$ image shows little structure but Ca K shows significantly more bright features than did the quiet Sun region, and they are well correlated with magnetic field strength (linear correlation coefficient of $64 \%$, rank coefficient 0.35 ). The brightest features in the 85 and $17 \mathrm{GHz}$ images coincide, and lie over a bright $\mathrm{Ca} \mathrm{K}$ feature in the south-east of the field but still within the coronal hole. The correlation coefficients between the $\mathrm{Ca} \mathrm{K}$ image and the $85 \mathrm{GHz}$ image are 0.26 (linear) and 0.31 (ranked), while between $\mathrm{Ca} \mathrm{K}$ and $17 \mathrm{GHz}$ they are 0.41 (linear) and 0.42 (ranked): we do not regard the difference between these correlations as significant.

Figure 6 shows overlays of contours of the $85 \mathrm{GHz}$ emission (left) and the longitudinal magnetic field (right) on the $\mathrm{Ca} \mathrm{K} \mathrm{im-}$ age for the coronal hole field. This overlay again emphasizes the general agreement between the $85 \mathrm{GHz}$ morphology and the $\mathrm{Ca} \mathrm{K}$ morphology (consistent with the fact that the ranked correlation coefficient of the two images is larger than the linear correlation coefficient). The boundary of the bright $85 \mathrm{GHz}$ emission matches the bright features in the Ca II $\mathrm{K}$ image very well. The ranked correlation coefficient between the magnetic field image and the $\mathrm{Ca}$ II $\mathrm{K}$ image is 0.35 , very similar to that between $\mathrm{Ca}$ II $\mathrm{K}$ and the $85 \mathrm{GHz}$ image. There are no deep depressions in the radio images of this field. The total flux in the $85 \mathrm{GHz}$ image is about $4.9 \mathrm{sfu}$ (not corrected for primary beam response), with a peak flux of order 0.10 sfu per beam corresponding to a brightness temperature range of $1700 \mathrm{~K}$.

\section{Sensitivity of millimeter interferometer data to oscillations}

A motivation for observing the solar chromosphere with a millimeter-wavelength interferometer is to be able to make images with high spatial resolution at high time resolution and thus to look for wave power. Due to the unusual way in which the 


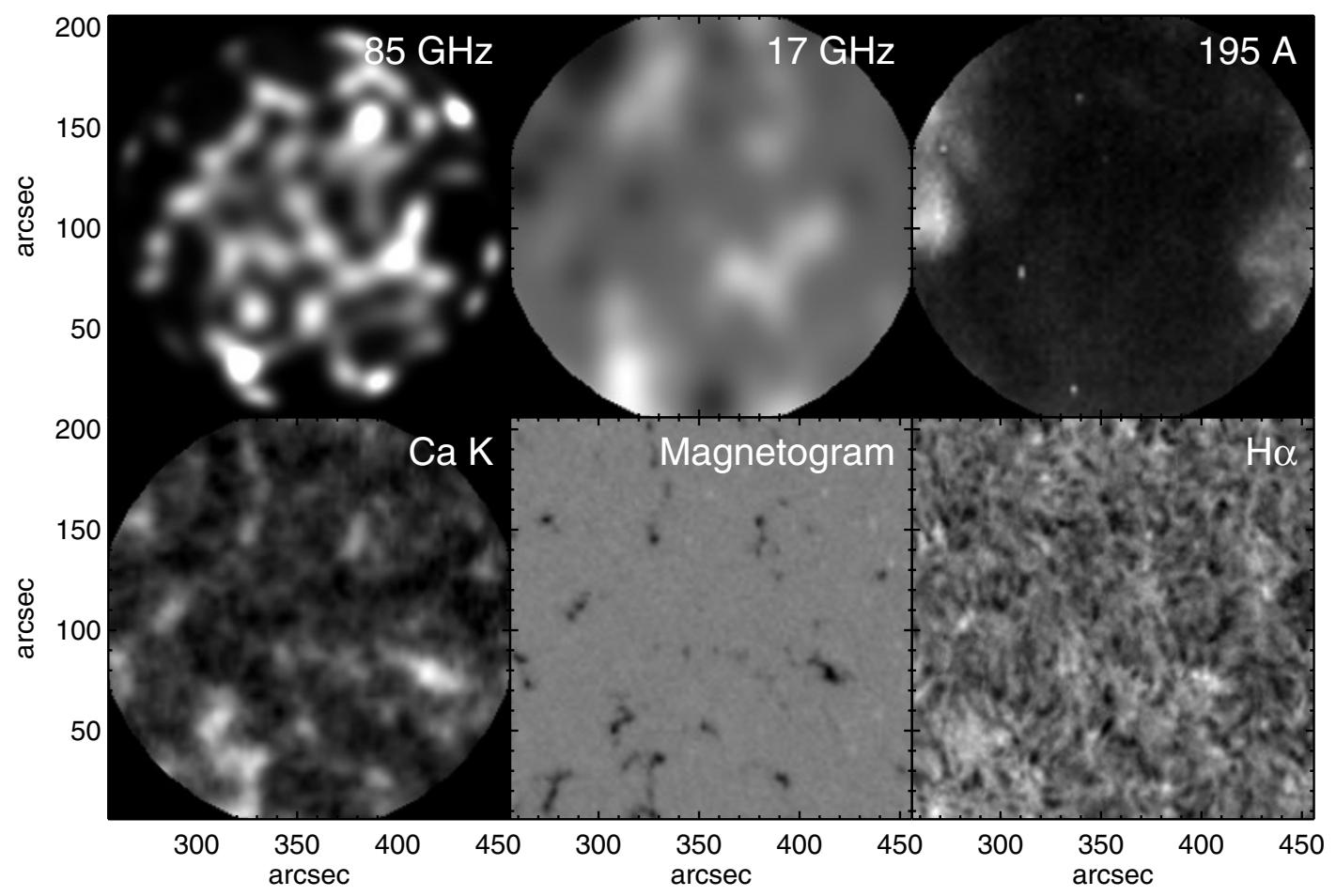

Fig. 5. Comparison of the coronal hole $(\mathrm{CH})$ field of view at a number of wavelengths in the same format as Fig. 2. The magnetogram display range is $\pm 300 \mathrm{G}$, at $17 \mathrm{GHz}$ the display range is 8500 to $12500 \mathrm{~K}$, and at $85 \mathrm{GHz}$ the display range is 0 to $2000 \mathrm{~K}$ (with correction for the primary beam response).

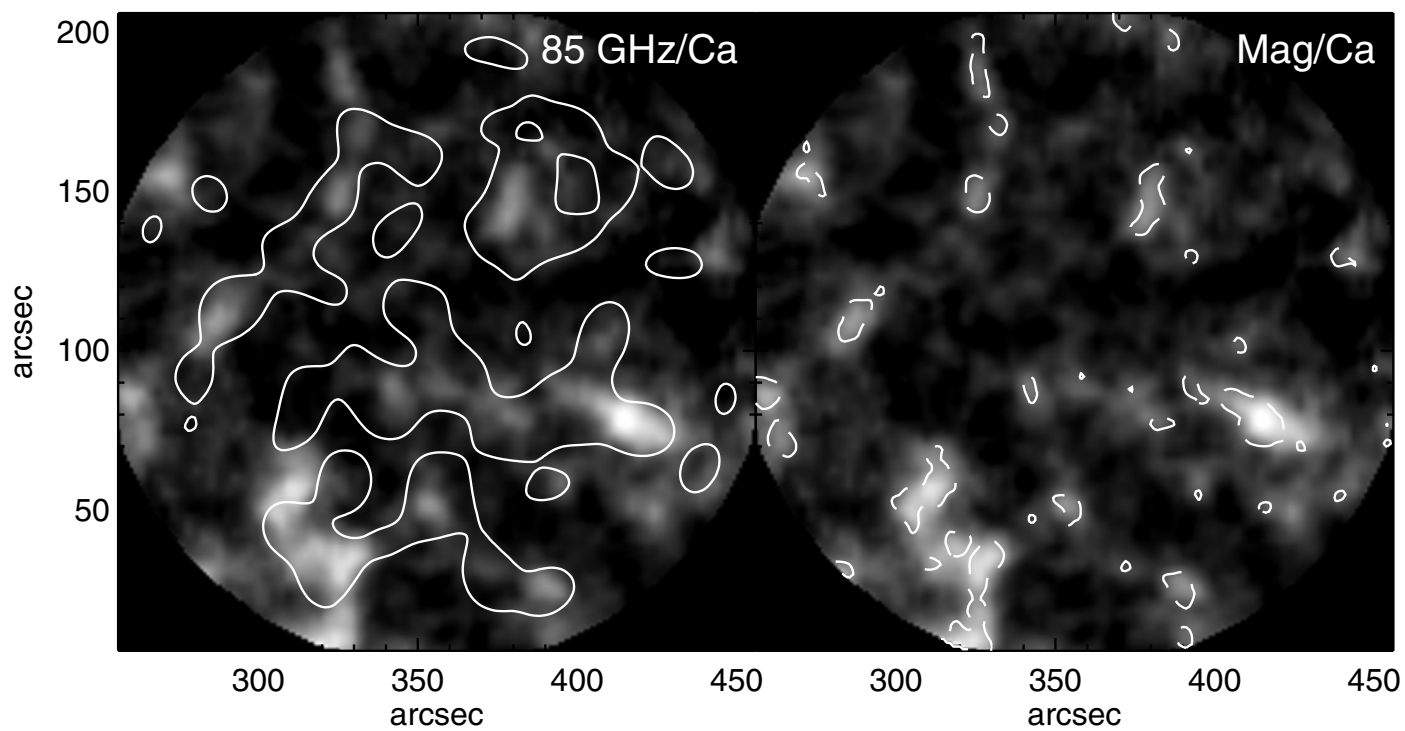

Fig. 6. Overlays of contours of the BIMA $85 \mathrm{GHz}$ emission (left) and the MDI longitudinal magnetic field (right) on the BBSO 16:37 UT Ca II K image (greyscale) for the coronal hole field of view. The millimeter contour is for the second active region scan at a brightness temperature of $600 \mathrm{~K}$, while the magnetic field contour is at $\pm 40 \mathrm{G}$.

snapshot BIMA images have to be derived (i.e., maximum entropy deconvolution with a default image based on the average over the whole time period), in a search for wave power it is important to understand the sensitivity of the imaging technique to waves. In Sect. 2 we briefly described tests of the sensitivity of the images to changes. In this section we specifically test for the ability to recover oscillations.

The tests were carried out as follows. The actual sequence of visibility data for a given target was modified by the addition of point sources whose amplitude varied sinusoidally in time (using the MIRIAD task uvmod). The data with and without the test source were then processed identically, as described above: dirty maps were inverted from the visibilities and deconvolution was carried out using maximum entropy with a default image. The final maps without the test source (i.e., the original BIMA data) were then subtracted from the corresponding maps made with data including the test source. In practice, we made the test somewhat more complex by adding two point sources at a time, one with a period of $290 \mathrm{~s}$ (five-minute oscillation) and an amplitude of 0.012 sfu that was $15 \%$ of the maximum in the mean image, and the other with a period of $185 \mathrm{~s}$ (three-minute oscillation) and an amplitude of 0.008 sfu that was 


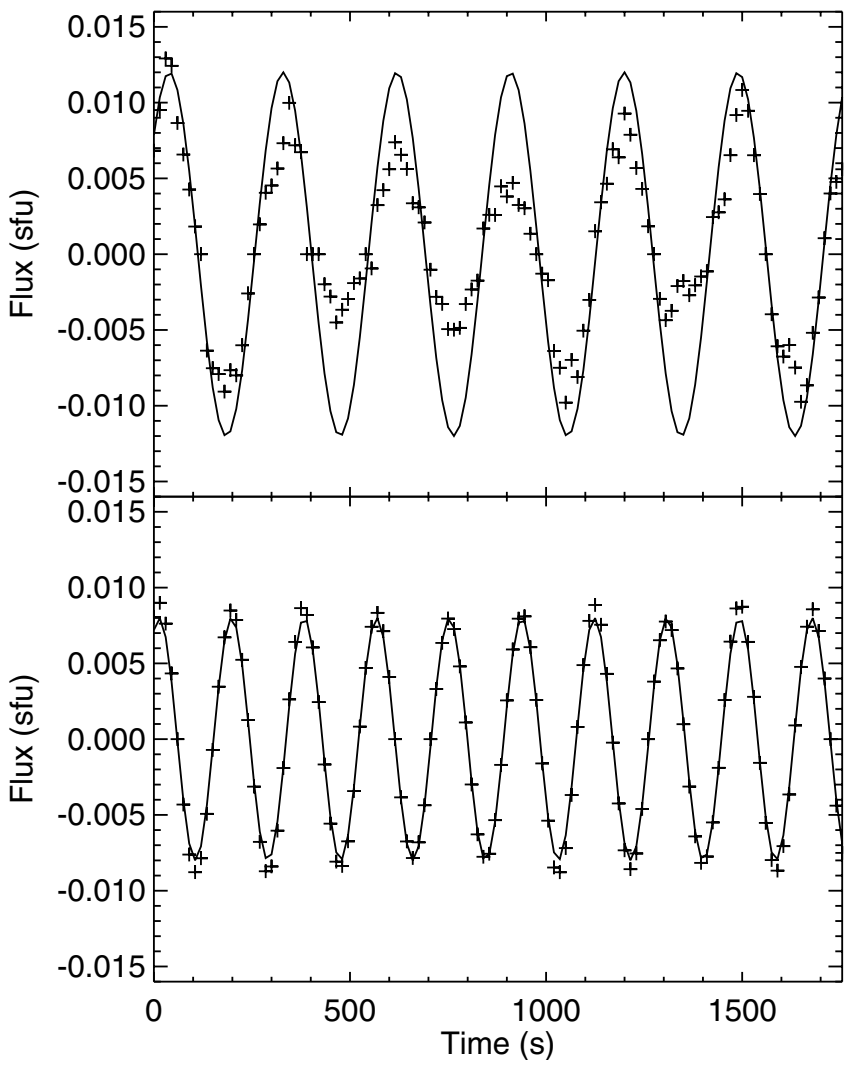

Fig. 7. Comparison of the recovery of the flux of the test oscillating source in two cases. In each panel the plus symbols are the fluxes recovered by fitting the difference between the deconvolved image with the test source and the deconvolved image without it, while the solid line represents the flux of the oscillating test source. The upper panel shows the results for a $290 \mathrm{~s}$ oscillation with an amplitude of $0.012 \mathrm{sfu}$ located in a region of low flux, while the lower panel shows the results for a $185 \mathrm{~s}$ oscillation of amplitude 0.008 in a region of higher flux.

just $10 \%$ of the maximum in the mean image. In the images these point test sources should appear as $10^{\prime \prime}$ features with brightness temperature amplitudes of 200 and $135 \mathrm{~K}$, respectively. Seven different test sequences were run, each consisting of 118 integrations $15 \mathrm{~s}$ apart using the second scan of the quiet-Sun target region, with the two sources in different locations each time. In each case, Gaussian fits to the difference images were made at the known locations of the test sources, limiting the size of the fitted source to be no bigger than about twice the restoring beam size.

The nature of the results can be seen in Fig. 7, where we compare the resulting light curves for one of the best recovered oscillations (lower panel, $185 \mathrm{~s}$ period) and one of the worst (upper panel, $290 \mathrm{~s}$ period) with the input test source fluxes. Even in the poorer case, the oscillation is clearly seen, while in the lower panel the agreement between the input fluxes and the recovered fluxes is extremely good. However, the fits always recovered sources that were spatially more extended than the model point source, indicating that maximum entropy deconvolution did not have enough information in the data to get the source dimensions correct. The recovered source dimensions were small in cases where the oscillations were well recovered but much broader in cases of poor recovery. In some cases the deconvolved test source did not resemble the model at all: for example, the test source located in the circular depression centered at coordinates $\left(-233^{\prime \prime},-53^{\prime \prime}\right)$ produced flux in the deconvolved maps that appeared in the bright ring surrounding the depression rather than in the depression itself, consistent with poor flux recovery at low flux values.

In Fig. 8 we present a summary of the overall test. In the left panel we show the locations of the test sources overlaid on the mean image for the period being considered. On the right panel we plot the fraction of the oscillation recovered (encircled plus symbols) as a function of the flux at the location of the test source in the average map. The fraction of the oscillation recovered here is defined as the quantity $\left(\sum_{i} S_{i} T_{i}\right) /\left(\sum_{i} T_{i}^{2}\right)$, where $S_{i}$ are the measured fluxes from the Gaussian fits to the difference images and $T_{i}$ are the input fluxes of the test source at that location; this quantity is unity for perfect recovery of the model fluxes, $S_{i}=T_{i}$, but if the $S_{i}$ measurements do not correlate with the $T_{i}$ values the product will be zero. The results are in agreement with the earlier tests: at locations where the mean flux is high the test source is recovered extremely well, whereas at locations with low mean fluxes recovery can be quite poor (although in no case do we fail to see the test source at all).

We also plot values for two other comparisons: in a given test we can create the above product using the "wrong" test source fluxes, i.e., multiplying the measured fluxes at the location of the $290 \mathrm{~s}$ source with the model fluxes for the $185 \mathrm{~s}$ source, and viceversa. These two quantities should be out of phase and thus on average cancel out: the triangle symbols in the right panel represent this quantity and show that this is indeed the case. Secondly, to investigate whether flux that is not recovered at the location of the test source is spread out over the rest of the map, we measured fluxes at the location marked by the square in the left panel of Fig. 8. This location has a mean flux that is $20 \%$ of the maximum in the average map. The squares in the right panel of Fig. 8 show the "fraction of the model flux recovered" at this location, i.e., the sums defined above where the $S_{i}$ values are measured at this location, for both the $290 \mathrm{~s}$ and $185 \mathrm{~s}$ test source $T_{i}$ values. This quantity is zero in the cases where the test source was well recovered, but can be quite large and often negative in the cases of poor recovery, suggesting that indeed the flux of the oscillating source can be redistributed over the image, but often out of phase, when the oscillation is located in a region of low mean flux.

The pattern in the upper panel of Fig. 7 actually suggests a reason for the poor recovery of test source flux at locations of low flux in the mean map. There is an asymmetry in the recovery of the positive and negative values of the model source: the positive values in general seem to be better recovered. This may be related to the positivity constraint of the maximum entropy algorithm used here: if the test source location becomes more negative than the rest of the field, then in order for it to be recovered while the positivity constraint is satisfied, all other pixels in the image need to have their flux values increased. In this case recovery of the model requires changes in many pixels to be correctly tracked by the deconvolution within the finite number of iterations of maximum entropy deconvolution typically chosen to reach a stable result within a reasonable time. By contrast, in the case where the test flux is positive or the underlying flux is quite large, the deconvolved image will only show changes at the location of the test source and relatively few pixels need to be changed, presumably making for a simpler deconvolution problem.

From these tests, we conclude that individual oscillating sources can indeed be recovered down to quite low amplitudes (certainly less than $10 \%$ of the contrast in the image, i.e., of order $100 \mathrm{~K}$ ) using the mapping technique for BIMA data applied here, provided that they lie in regions where the mean flux is not too low. It seems plausible that heating power should 

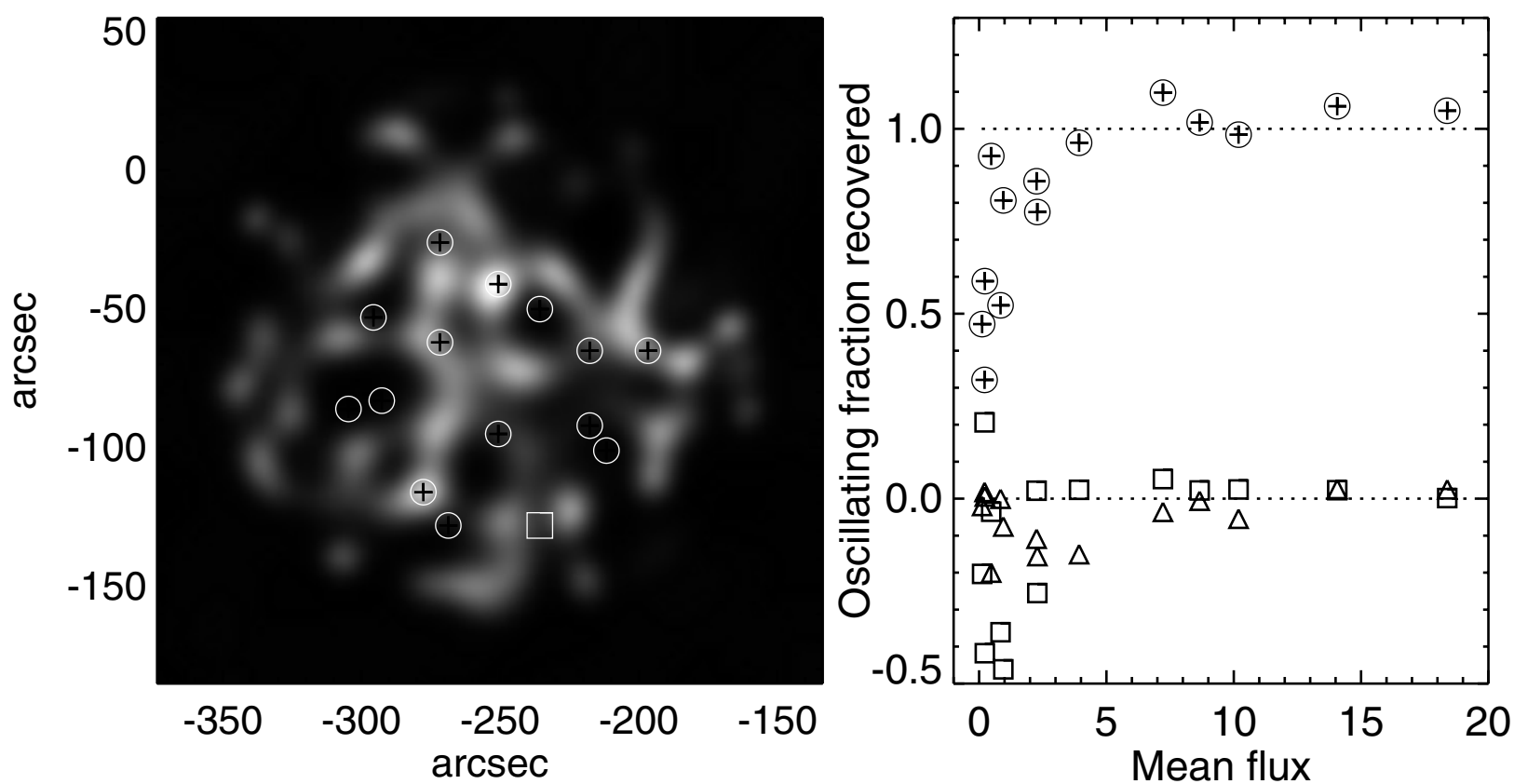

Fig. 8. Tests of the recovery of oscillating test sources in the BIMA observations. The left panel indicates 14 locations (black plus symbols surrounded by white circle) where oscillating sources were placed in seven separate simulations (two sources per simulation, one with a $290 \mathrm{~s}$ period and the other with a $185 \mathrm{~s}$ period) of the second scan of the quiet Sun (average image shown). The right panel shows the fraction of the test source flux recovered for each of the 14 sources (plus symbols surrounded by circles), defined as $\left(\sum_{i} S_{i} T_{i}\right) /\left(\sum_{i} T_{i}^{2}\right)$ where $S_{i}$ are the recovered flux values and $T_{i}$ are the input test source fluxes, so that a value of 1.0 corresponds to perfect recovery of the input source. For comparison, the triangles are the same quantity for the 14 locations but swapping the $185 \mathrm{~s}$ and the $290 \mathrm{~s}$ test fluxes: the $S_{i}$ and $T_{i}$ values then should be out of phase with each other and cancel, as is found. The squares represent the same quantity but for the location marked by a square in the left panel, where no test source was located: in each of the seven tests we extracted the light curve at this location and constructed the product above for both the $290 \mathrm{~s}$ and $185 \mathrm{~s}$ oscillations, resulting in the 14 values plotted. In each case the square symbol is plotted underneath the corresponding test source product.

predominantly lie in regions where the mean flux is higher since we expect that regions of strong heating are likely to be bright in the BIMA images. These tests do not, of course, truly represent the likely appearance of oscillation power in the millimeter images, but the test sources used here represent only a small fraction of the total flux present (in these tests, the two sources total about $0.5 \%$ of the 4 sfu of flux in the deconvolved images), so the results do indicate that quite small levels of oscillation power can be detected with the technique described.

However, if oscillation power is present everywhere in the image simultaneously then the limited number of baselines available to make the snapshot images will limit our ability to reconstruct oscillation power in many resolution elements simultaneously. We have carried out a test in which 8 model point sources are added to the visibility data for a single snapshot at random locations and then the resulting snapshot data are deconvolved using the same default model as before. As expected based on the arguments given above, we find that some of the sources are well recovered in the deconvolved map, while those located in regions of weak flux are not and their flux appears redistributed, spread as diffuse emission across other brighter locations in the image. On this basis, we argue that oscillation power present in the target region does not seem to be lost by the deconvolution approach we adopt here, but it may not appear in the correct location since the tests indicate that power located in regions of low flux may not be accurately recovered. Bright oscillating sources in locations of steady bright emission, on the other hand, may be well represented in the final maps. This is a limitation of the technique we employ (the only technique we found which produced satisfactory results, limited by the small number of antennas in the array and the low-contrast nature of the brightness distribution), and better results will require images from an interferometer with many more elements, such as the Atacama Large Millimeter Array (ALMA). We briefly investigate what oscillation properties the BIMA image sequences display in the following section.

\section{Oscillation power in the millimeter images}

Based on the analysis given in the preceding section, we expect that if the oscillation power is dominated by a small number of features located in regions of bright emission then it should be well represented by these data, but if significant oscillation power resides in locations that are faint in the daily average image used as a model for the time-dependent analysis then it is likely to be redistributed into regions of bright emission and contaminate the oscillation spectra there. In this section we investigate the oscillation properties shown by these data using standard analysis approaches, bearing in mind the limitations imposed by the deconvolution method. A more detailed analysis and comparison with models is presented in a companion paper by Loukitcheva et al. (2006).

\subsection{Analysis technique}

After the procedures of image restoration and elimination of poorer data we are left with nine uninterrupted 30-min sequences of snapshot images (in units of brightness temperature): 3 successive quiet-Sun scans (QS2, QS3, QS4), 3 active region scans (AR2, AR3, AR4) and 3 coronal hole scans (CH1, CH2, CH4). All images were corrected for the primary beam response and 
truncated in a 72" radius field of view. For the analysis of the radio oscillations we employed two standard techniques: Fourier power spectra, and wavelet transforms. Comparison of results from two independent techniques gives us more confidence in our conclusions. Power spectra were obtained by applying the Fast Fourier Transform algorithm including a 10\% cosine apodisation. Before processing, the long term evolution in each pixel was removed by subtracting a third order polynomial fit to the brightness temperature time series. No other filtering was carried out in order to preserve the original oscillatory power. The statistical significance of the oscillations for individual and spatially averaged time series was estimated using the prescription of Groth (1975), depending on the level of the (white) noise in the power spectra. (The actual noise characteristics are difficult to assess given the complex processing undergone by the data, but for simplicity we will proceed as if white noise is the appropriate assumption.) Only power above the $99 \%$ significance level was considered significant.

The Nyquist frequency of the image sequences is $33 \mathrm{mHz}$, corresponding to a period of $30 \mathrm{~s}$, and due to the discrete sampling any period in the signal shorter than $30 \mathrm{~s}$ will be aliased. The duration of the significant oscillations as well as the evolution of their periods were studied by means of a wavelet analysis. As a mother wavelet we use the complex valued Morlet wavelet that consists of a plane wave modulated by a Gaussian. For the wavenumber $k$, which describes the number of oscillations within the wavelet itself, we employ $k=6$. In the wavelet transform we adopted a total of 41 scales ranging from $30 \mathrm{~s}$ up to 960 s. Significance levels of $99 \%$ were calculated assuming the global wavelet spectrum as a background spectrum, following the prescription of Torrence \& Compo (1998). We consider only time series showing brightness variations exceeding the noise level, which corresponds to $100 \mathrm{~K}$ in brightness temperature (Sect. 3.3).

\subsection{Oscillation power in different regions}

Before analyzing the oscillatory behavior at individual locations we construct the power spectra for the spatially averaged time series. In order to be suitable for comparison with the results of single dish observations the averaging was done over all spatial locations within fields of view of different radii in the range $20-72^{\prime \prime}$. To build the time series equivalent to those obtained using typical single-dish beam sizes the correction for the Gaussian shape of the beam was applied before averaging of individual time series. For spatial averaging within a $20^{\prime \prime}$ field of view the corresponding power spectra of the QS2, AR2 and $\mathrm{CH} 2$ time series, acquired when atmospheric phase fluctuations were minimal, are depicted in Fig. 9 by solid, dashed and dotted curves, respectively. The averaged quiet Sun time series demonstrates variations of brightness temperature corresponding to an rms of $21 \mathrm{~K}$, while for the $\mathrm{AR}$ and $\mathrm{CH}$ targets the variations are each about $20 \mathrm{~K}$. Figure 9 clearly shows that oscillatory behavior is present in all the target regions. For the QS power spectrum the power peaks at $6.8 \mathrm{mHz}$ (corresponding to a period of $147 \mathrm{~s}$ ), $2.3 \mathrm{mHz}(435 \mathrm{~s})$ and $1.4 \mathrm{mHz}(714 \mathrm{~s})$ are statistically significant. In the $\mathrm{AR}$ and $\mathrm{CH}$ spectra the significant peaks are at $3.8 \mathrm{mHz}$ (263 s) and $2.9 \mathrm{mHz}(345 \mathrm{~s})$. The averaged $\mathrm{CH}$ time series shows additional significant peaks at frequencies of $2 \mathrm{mHz}(500 \mathrm{~s})$ and $1.1 \mathrm{mHz}(909 \mathrm{~s})$ : however, $1.1 \mathrm{mHz}$ is the longest period to which the 30-min data sequences are sensitive and data at the longer periods are affected by the detrending method used, so the exact nature of this peak is unclear but the presence of power at longer periods is robust.

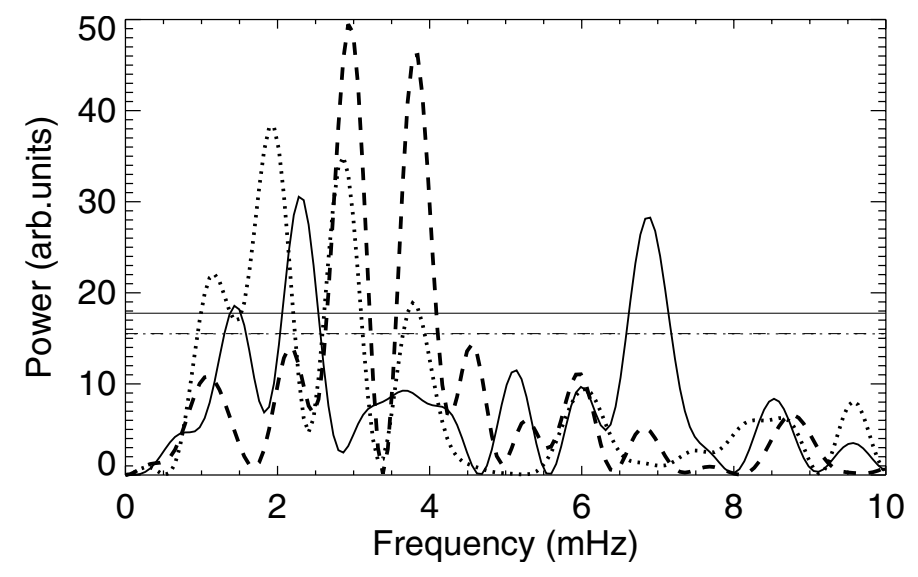

Fig. 9. Power spectra for the spatially averaged QS2 (solid curve), AR2 (dashed curve) and $\mathrm{CH} 2$ (dotted) data within a 20" radius field of view. Frequencies above $10 \mathrm{mHz}$ show no significant peaks and are therefore omitted for clarity; other details are given in the text. The horizontal lines are the 99\% significance thresholds for the three power spectra, according to line type (the dashed AR and dotted $\mathrm{CH}$ thresholds overlap).

The amplitudes of the intensity variations in the averaged time series are higher than the results of Kislyakov et al. (1996), who reported significant oscillations at several frequencies in the range of $1-7 \mathrm{mHz}$ with amplitudes of $2-10 \mathrm{~K}$ in the radio emission of the quiet and active Sun at the wavelength of $3.3 \mathrm{~mm}$ within a beam of $40^{\prime \prime}$. Similar results were obtained for the quiet-Sun continuum in the wavelength range of 300-800 $\mu \mathrm{m}$ by Lindsey \& Kaminski (1984), who found local intensity variations within the beam of $48^{\prime \prime}$ diameter at frequencies in the range 3-7 $\mathrm{mHz}$ with rms brightness temperatures of order $5 \mathrm{~K}$.

Using average power spectra may not be the most robust way of revealing differences between the oscillation properties of the three types of regions. Moreover, Fig. 9 is not completely representative of the power spectra corresponding to each individual point, which show a considerable scatter, with, e.g., relative amplitude of power peaks changing significantly from one point to the next. Also, the frequency of the peak power can shift slightly from one point to another. A more robust comparison of the frequency distribution in the intensity oscillations of the quiet Sun and active region is obtained if we determine the frequency (period) at which the maximum power is found for the power spectrum of each pixel containing significant peaks. The corresponding distributions $N(P)$ derived for $\mathrm{QS}$ and AR data, which describe the number of pixels having maximum power at period $P$, are shown in Fig. 10, i.e. this figure shows the relative occurrence of different dominant periodicities in individual pixels in the field of view. For the quiet Sun the maximum power, depicted by the solid line in Fig. 10, is distributed over a broad range between 150 and $1000 \mathrm{~s}$, with a distinct maximum in the distribution at 200-350 s. The histogram for the AR (dashed line in Fig. 10), also reveals a broad range of frequencies, but with a clear maximum in the histogram at about $800 \mathrm{~s}$. In addition to the dominant period band of 500-850 s the AR data also show smaller peaks at shorter periods of 200 and $300 \mathrm{~s}$, although the statistics are not adequate for us to assert that these are really separate peaks. Figure 10 clearly suggests that in the active region target longer periods dominate over short periods (although as noted earlier the actual distribution of power at long periods is affected by the data analysis and the limited sampling on longer timescales), while in the quiet Sun there is a wide distribution of power with a peak at short periods. The broad histograms are 


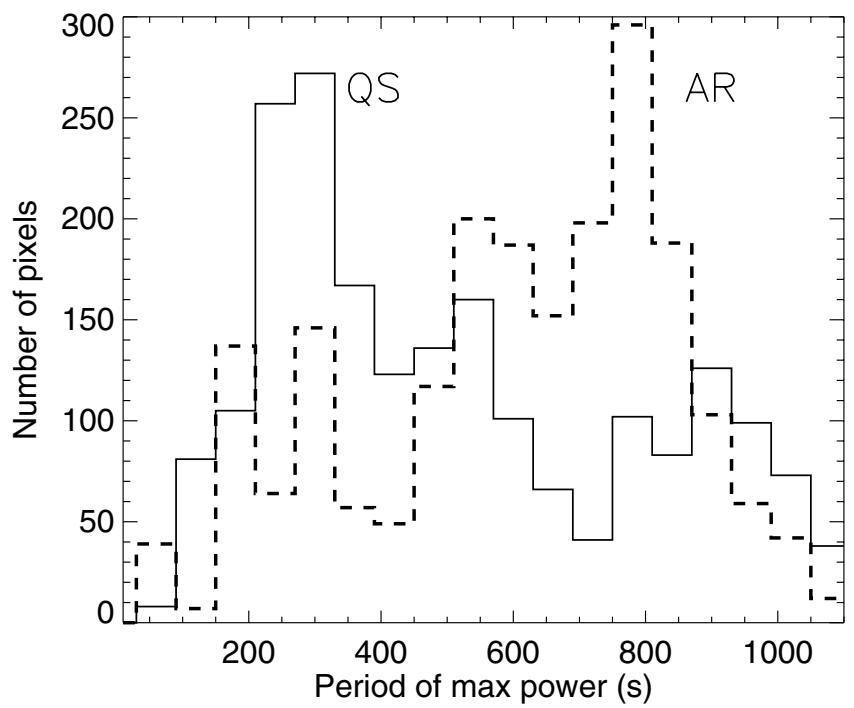

Fig. 10. Histograms of the period of maximum power for the QS (solid line) and AR data (dashed line). For each pixel the period with most significance in the power spectrum is determined exactly as described in Sect. 6.1, and at this period the pixel is included in the distribution. Individual pixels in the millimeter images are not statistically independent, and it is the relative, not the absolute, number of pixels that is important. There are about 10 pixels per resolution element.

illustrative of the fact that the dominant peak can be located at any frequency within a given range, making the average spectrum susceptible to the limited statistics.

\subsection{Distinction between network and cells}

Observations in the cores of the chromospheric spectral lines (e.g. the Ca II K line) reveal a distinct difference in the oscillation frequencies of quiet sun network (NW) and supergranulation cell interiors. The latter are often also referred to as the internetwork (IN). The network is dominated by oscillations with periods of $5 \mathrm{~min}$ and longer, while the internetwork regions display enhanced power in the 3 min range (see, e.g. Rutten \& Uitenbroek 1991; Lites et al. 1993). This difference is thought to be produced by the differing magnetic structure in the network (strong, mainly vertical fields) and the internetwork (weak, partly horizontal fields, in a canopy-like configuration). We now investigate whether the oscillation properties seen in the quiet Sun millimeter image sequences also show this distinction, bearing in mind the possibility discussed above that power present in regions of low brightness can appear to leak into brighter regions due to the limitations of the deconvolution technique. Each observed field was divided into internetwork (IN) pixels, network $(\mathrm{NW})$ pixels or an intermediate region based on masks constructed from the BBSO Ca II K images and time-averaged radio images for each data set. A pixel was assigned to represent internetwork if its calcium intensity was below the peak of the $\mathrm{Ca}$ II $\mathrm{K}$ intensity histogram and its mean radio brightness was below half of the maximum brightness of the time-averaged QS image. To define the network a Gaussian fit was applied to the calcium intensity histogram. If a pixel displayed an intensity value lying more than $1 \sigma$ above the intensity of the maximum of the Gaussian fit and its mean radio brightness was above half of the maximum brightness of the time-averaged image, then it was assigned to the network. We found it necessary to use this double criterion involving both $\mathrm{Ca}$ II K and millimeter wavelength brightness. The advantage of employing Ca II $\mathrm{K}$ is that it shows a strong contrast between network and internetwork. However, due to the significantly different spatial resolutions of the calcium and millimeter data sets it is important to consider also the latter. Finally, only pixels defined as network (internetwork) in all 3 quiet Sun data sets were considered when creating the network (internetwork) mask.

Spatially resolved BIMA IN and NW time series exhibit a wide variety of power spectra with the peak of maximum power varying from the 3-min range up to $10 \mathrm{~min}$ and longer. Nevertheless, among them one can find examples of time series which manifest power spectra known from other observations to be typical for IN and NW locations. We show the results of the Fourier and wavelet analysis for these "typical" internetwork and network locations in Figs. 11 and 12, respectively. Inspection of the time series in Figs. 11a and 12a suggests the presence of quasi-periodic variations with amplitudes larger than is expected from pure noise. The chosen time series correspond to the spatial locations $\left(-311^{\prime \prime},-44^{\prime \prime}\right)$ and $\left(-269^{\prime \prime},-92^{\prime \prime}\right)$ in the QS2 integration. For the internetwork location the dominant power peak is at about $5.2 \mathrm{mHz}$ (corresponding to a period of $192 \mathrm{~s}$ ) with a secondary peak at $3.0 \mathrm{mHz}$ (period of $333 \mathrm{~s}$ ), whereas in the network location the $5-\mathrm{min}(3.2 \mathrm{mHz})$ oscillations are the strongest in addition to significant power at $2.2(455 \mathrm{~s})$ and $4.5 \mathrm{mHz}$ (222 s). Figures 11c and 12c, depicting the wavelet spectra, in principal allow the duration of significant oscillations at a given location to be determined. In practice, the relatively short length of the time series limits its usefulness. In the wavelet spectrum figures the darker contour regions show the location of the highest power. Cross-hatched regions indicate the limit of the cone of influence (COI), outside which edge effects become important (see Torrence \& Compo 1998). Nonetheless, Fig. 12c suggests that the period of maximum power for the network oscillations increases from about $200 \mathrm{~s}$ initially to $300-500 \mathrm{~s}$ later in the scan. The internetwork location displays a wavelet spectrum of quite different character (Fig. 11c), with the most powerful oscillations remaining at a period of about 180-200 s throughout, accompanied by oscillations with the power maximum at longer periods of about 300-350 s. Several periodic events are recorded in the high frequency range of 50-150 s and last for only short periods in both IN and NW wavelet spectrum plots.

It is instructive to compare the oscillation frequency distributions for pixels representing internetwork and network and make a figure similar to Fig. 10 to look for differences between the non-magnetic and magnetic quiet Sun. The corresponding histograms of the period of maximum power for the quiet Sun internetwork and network are depicted in Fig. 13 by solid and dashed lines, respectively. The data have been divided by the number of pixels to make the histograms directly comparable: 780 time series from 3 QS data sets were used to construct the IN histogram and only 83 time series represent network locations in the histogram. In the period range of 200-400 s the histograms show comparable amounts of power, but the peak of the IN histogram is at shorter periods (210-270 s) than the NW histogram peak (270-330 s). From the prominent NW histogram peak at the period of 510-570 s it is seen that the network has a greater oscillatory power at longer periods. The relative positions of the peak power in the IN and NW data sets found here agrees with the results of McAteer et al. (2004), who analyzed ultraviolet chromospheric oscillations by means of a wavelet-based technique. Those authors demonstrate that the most frequent internetwork oscillations occur at periods of 205-309 s, while for the network the oscillations are commonly present in the period range of 231-346 s. However, we emphasize that because it is difficult to assess the uncertainty in the spatial locations of the peaks in 

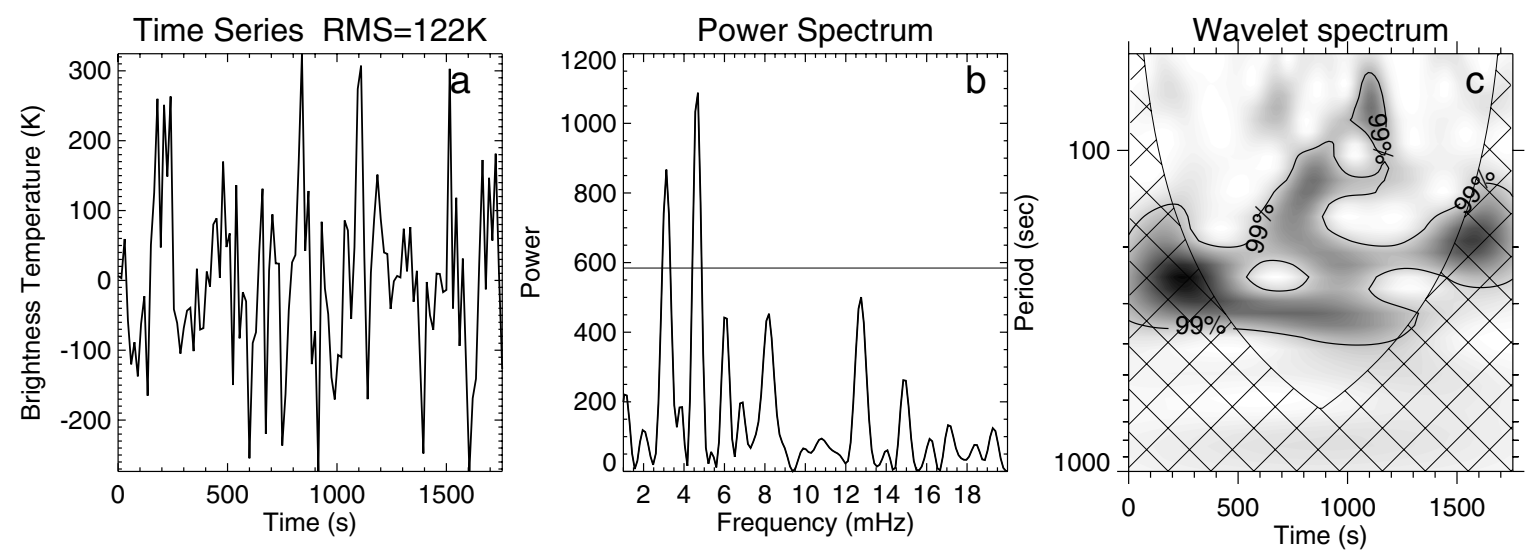

Fig. 11. Oscillations at the internetwork location $\left(-311^{\prime \prime},-44^{\prime \prime}\right)$ of the QS2 integration. a) Intensity time series with the long-term trend subtracted. b) Corresponding Fourier power spectrum as a function of frequency. c) Wavelet power spectrum as a function of time and period (grey scale). Cross-hatched regions indicate the cone of influence, where the nearby boundary affects the reliability of the results. Thin solid lines in b) and c) represent the $99 \%$ significance level of oscillations.
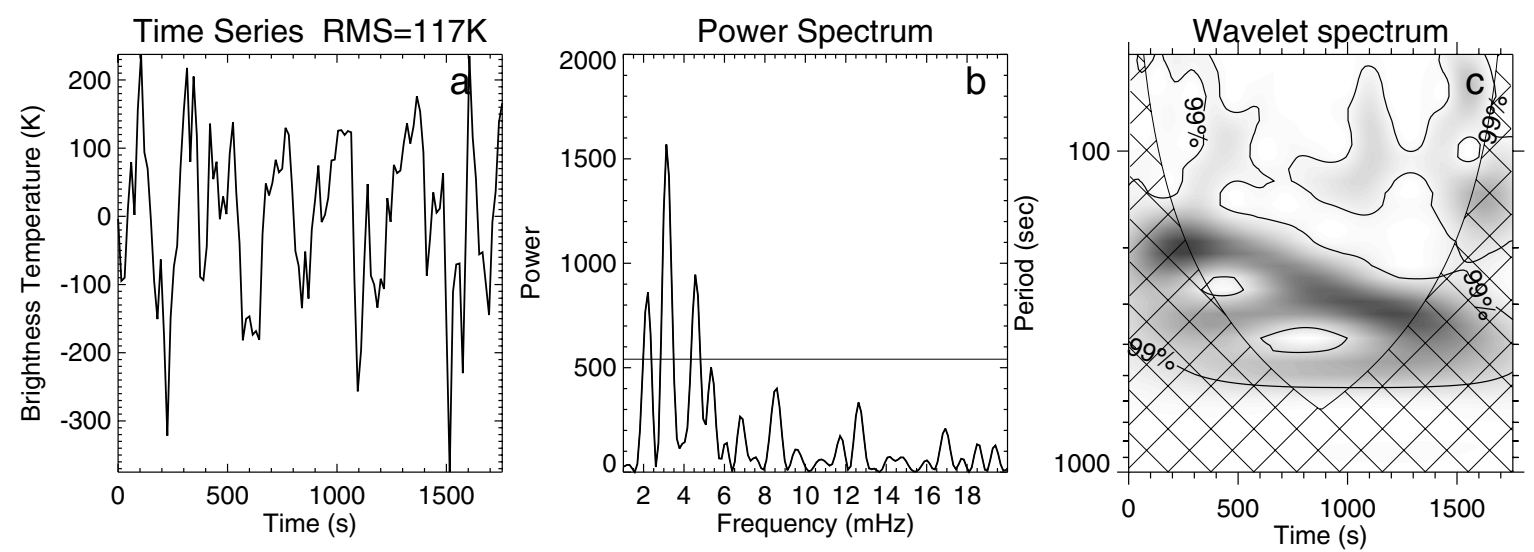

Fig. 12. The same as Fig. 11 for a network location $\left(-269^{\prime \prime},-92^{\prime \prime}\right)$ of the QS2 integration.

the BIMA data due to the possibility that oscillating power from regions of weak flux is redistributed across the image, we cannot make a very strong statement about this agreement and further studies are needed to confirm it. Based on the earlier tests, we might expect a transfer of oscillation power from internetwork locations that are weaker in the overall image to brighter network locations. The complete absence of the 540 s network peak in the internetwork histogram certainly suggests that there is little leakage of oscillation power from the bright regions of the image to the fainter pixels.

For comparison with Figs. 11 and 12 we plot in Fig. 14 the spatially resolved time series and the results of the Fourier and wavelet analysis for the location (135", 177") from the AR2 time series. The Fourier spectrum shows a peak at $3.3 \mathrm{mHz}$ (period of $303 \mathrm{~s}$ ) with secondary peaks at $1.7,4.2$ and $6.2 \mathrm{mHz}$. Five-minute and 10-min oscillations are present during the whole observation, and there is short-period power with a period that seems to increase from 2 to 4 min over the duration of the scan. Not surprisingly, the active region power spectrum more closely resembles the bright-pixel network spectrum of Fig. 12 than the fainter-pixel internetwork spectrum of Fig. 11.

\section{Summary}

We present the first high-resolution two-dimensional maps of the solar chromosphere made with an interferometric array at millimeter wavelengths and use the data to investigate the

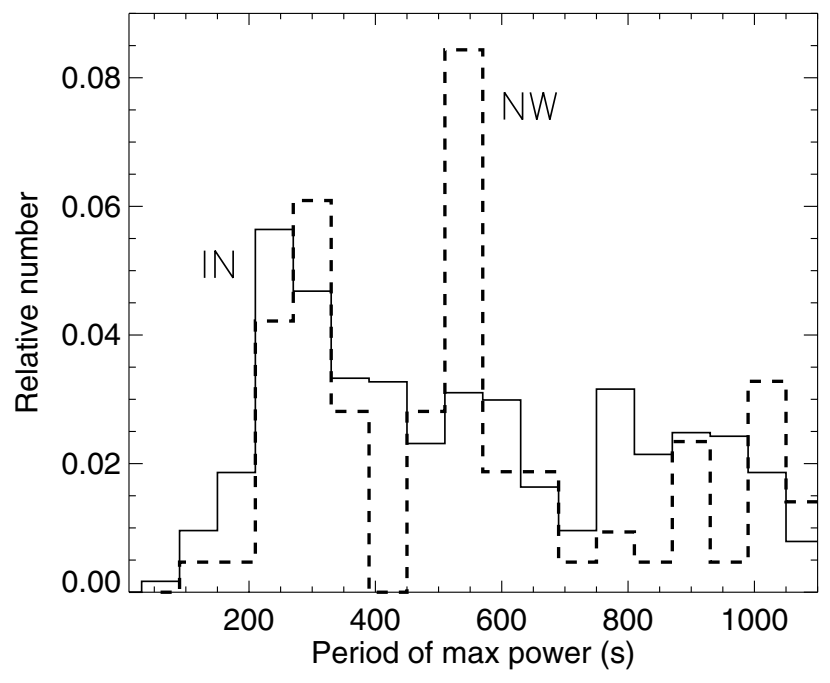

Fig. 13. Histograms of the period of maximum power for the quiet Sun internetwork (solid line) and network (dashed line). For each pixel the most significant power is determined and at this period the pixel is included in the distribution. The distributions are divided by the number of pixels.

potential for determining spatially-resolved power spectra of features within the solar atmosphere. Because of the lowcontrast nature of the Sun's brightness distribution at millimeter 

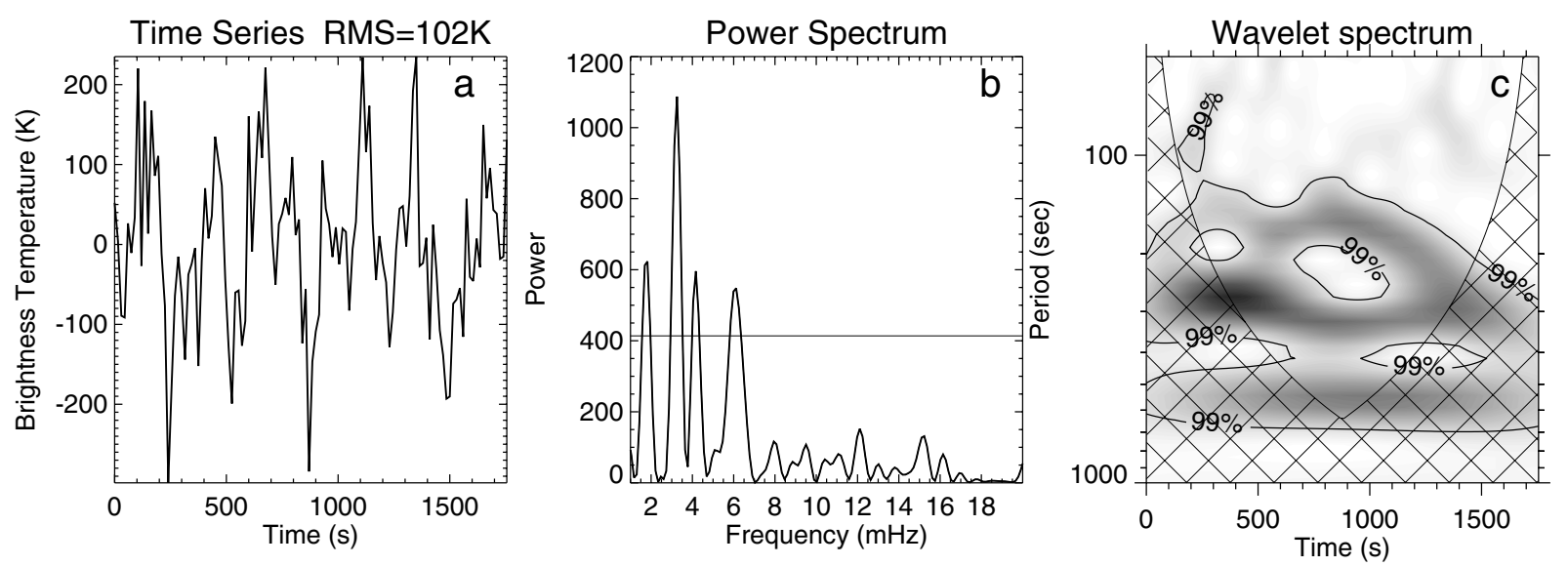

Fig. 14. The same as Fig. 11 for the AR2 location (135", 177").

wavelengths, we find that the conventional "CLEAN" deconvolution technique is unsatisfactory for making snapshot maps of the solar atmosphere but that maximum-entropy deconvolution is successful, provided that one uses a default image based on a long integration that contains enough information to reconstruct the spatial brightness distribution. This technique can only be successful if the underlying brightness distribution does not change completely on timescales shorter than a few hours, which one might fear would happen in a completely turbulent chromosphere. One of our results is that this is not the case: as is true of, e.g., Ca II K line observations of the chromospheric network, the millimeter brightness distribution is dominated by features that live for at least a number of hours, so that our technique for snapshot imaging only requires the deconvolution of small changes in the brightness distribution.

We carried out extensive tests of the sensitivity of the data to oscillation power and find that it can see sources with intensity variation amplitudes less than $10 \%$ of the brightest feature in the field, as long as the source is located where there is significant flux in the default image. We explain this result as an artefact of the positivity constraint employed in most maximum-entropy deconvolution algorithms: the zero level in the default image is set by the coolest feature in the field of view, and an oscillating source located in a region of weak emission that becomes negative can cause the effective zero level of the brightness distribution to change. The positivity constraint then requires deconvolution to adjust the flux in every pixel upwards from its value in the default image by the necessary amount: changing the flux in so many pixels requires more changes to the image than we have information available in a snapshot observation, and so deconvolution can fail. This is a limitation on our technique, and may lead to oscillation power that belongs in regions of weak emission showing up in regions of bright emission instead. Unfortunately the one readily available MEM program without a positivity constraint, UTESS in the AIPS package, failed to converge to a suitable solution even for the entire day's data.

Having established the viability of our technique for measuring wave power with these limitations, we use the best continuous 30-min data sets for the quiet-Sun and active region targets at $85 \mathrm{GHz}$, with a spatial resolution of $10^{\prime \prime}$, to study the spatially resolved properties of oscillation power in the millimeter data. Fourier and wavelet analysis reveals the presence of intensity oscillations with rms brightness temperatures of 50$150 \mathrm{~K}$ in the frequency range $1.5-8 \mathrm{mHz}$. In general, our analysis shows that at the chromospheric levels which contribute to radio emission at $85 \mathrm{GHz}$, active and quiet regions have different power spectra. The observations reveal a tendency toward short period oscillations in the quiet Sun and longer periods in the active region, which is in good agreement with the results obtained at other wavelengths. However, the data suggest a strong scatter in the dominant periods of wave power from one spatial position to another. Most of the oscillations are not steady harmonic waves but wave trains of finite duration lasting for typically 1-3 wave periods. The presence of short-lived wave trains, which can occur at somewhat different frequencies, may explain the variation from one spatial location to another. Longer sequences of images would increase the accuracy of these results.

The general difference in behavior between quiet and active regions is mirrored by internetwork and network regions in the quiet Sun, with the latter having a tendency to exhibit longer period oscillations. The spatial resolution of the observations of $10^{\prime \prime}$ probably hinders a clean separation between cell interiors and network since it is of the same order as the size of a supergranule (20-30"). Therefore, at many spatial points both network and internetwork areas contribute to the power spectrum. Further, the limitations of the MEM deconvolution mean that we might expect to see power that belongs in the weaker cell interior locations showing up in the brighter network pixels. We are not able to resolve individual oscillating elements and thus the direct comparison with the models, e.g. of Carlsson \& Stein (1992, 1995, 1997), see Loukitcheva et al. (2004), and of Wedemeyer et al. (2004), is not straightforward and needs an adequate description of the influence of atmospheric phase and deconvolution errors and a better knowledge of the predictions of the models. These topics are pursued in the companion paper by Loukitcheva et al. (2006).

On the whole, our work shows that high resolution millimeter wave observations are a promising diagnostic of chromospheric structure and dynamics. Observations at different millimeter wavelengths (e.g., 1 and $3 \mathrm{~mm}$ ) with arrays such as the Combined Array for Research in Millimeter-wave Astronomy (CARMA) and ALMA that contain many more antennas should result in much better understanding of the propagation of these waves. Our results clearly shows the need for further observations of this type with longer sequences and if possible higher spatial resolution.

Acknowledgements. Extensive discussions with the referee led to improvements in the paper. Millimeter-wavelength astronomy at the University of Maryland is supported by the National Science Foundation under grant numbers AST-0228974 and 0540450. Solar research at the University of Maryland is supported by NSF grant ATM 02-33907 and NASA grants NAG 5-10175, 
NAG 5-12860 and NAG 5-11872. SW thanks Dick Plambeck and Douglas Bock for installing the pellicles used in solar calibration of BIMA, and Rick Forster and the staff at BIMA for their help with solar observing. We gratefully acknowledge the open-data policies of NoRH, the NASA satellite TRACE and the ESA/NASA satellite SOHO and their instrument teams which make multi-wavelength research such as this much easier. Wavelet software was provided by $\mathrm{C}$. Torrence and G. Compo, and is available at the URL http://paos.colorado.edu/research/wavelets.

\section{References}

Bastian, T. S., Ewell, M. W., \& Zirin, H. 1993a, ApJ, 418, 510

Bastian, T. S., Ewell, M. W., \& Zirin, H. 1993b, ApJ, 415, 364

Bastian, T., Dulk, G., \& Leblanc, Y. 1996, ApJ, 473, 539

Beckman, J. E., Lesurf, J. C. G., \& Ross, J. 1975, Nature, 254, 38

Belkora, L., Hurford, G. J., Gary, D. E., \& Woody, D. P. 1992, ApJ, 400, 692

Benz, A. O., Krucker, S., Acton, L. W., \& Bastian, T. S. 1997, A\&A, 320, 993

Buhl, D., \& Tlamicha, A. 1970, A\&A, 5, 102

Carlsson, M., \& Stein, R. F. 1992, ApJ, 397, L59

Carlsson, M., \& Stein, R. F. 1995, ApJ, 440, L29

Carlsson, M., \& Stein, R. F. 1997, ApJ, 481, 500

Cornwell, T. J. 1988, A\&A, 202, 316

Cornwell, T., \& Braun, R. 1989, in Synthesis Imaging in Radio Astronomy, ed.

R. A. Perley, F. R. Schwab, \& A. H. Bridle (San Francisco: Astron. Soc. Pacific), Conf. Ser., 6, 167

Erskine, F. T., \& Kundu, M. R. 1982, Sol. Phys., 76, 221

Ewell, Jr., M. W., Zirin, H., Jensen, J. B., \& Bastian, T. S. 1993, ApJ, 403, 426

Fontenla, J. M., Avrett, E. H., \& Loeser, R. 1990, ApJ, 355, 700

Fontenla, J. M., Avrett, E. H., \& Loeser, R. 1991, ApJ, 377, 712

Gary, D. E., Zirin, H., \& Wang, H. 1990, ApJ, 355, 321

Groth, E. J. 1975, ApJS, 29, 285

Gu, Y., Jefferies, J. T., Lindsey, C., \& Avrett, E. H. 1997, ApJ, 484, 960

Hagen, J. P., Swanson, P. N., Haas, R. W., Wefer, F. L., \& Vogt, R. W. 1971 Solar Phys., 21, 286

Horne, K., Hurford, G. J., Zirin, H., \& de Graauw, T. 1981, ApJ, 244, 340

Irimajiri, Y., Takano, T., Nakajima, H., et al. 1995, Sol. Phys., 156, 363

Janssen, M. A., Olsen, E. T., \& Lang, K. R. 1979, ApJ, 228, 616

Kislyakov, A. G., Nosov, V. I., \& Tsvetkov, L. I. 1996, Izvestiya KRAO, 93, 47

Kopp, G., Lindsey, C., Roellig, T. L., et al. 1992, ApJ, 388, 203

Kosugi, T., Ishiguro, M., \& Shibasaki, K. 1986, Publ. Astron. Soc. Jpn, 38, 1

Kundu, M. R. 1970, Sol. Phys., 13, 348

Kundu, M. R. 1971, Sol. Phys., 21, 130
Kundu, M. R., \& Schmahl, E. J. 1980, A\&A, 90, 192

Kurths, J., Aurass, H., Urpo, S., \& Pohjolainen, S. 1988, A\&A, 191, 359

Labrum, N. R. 1978, Proc. Astron. Soc. Aust., 3, 256

Labrum, N. R., Archer, J. W., \& Smith, C. J. 1978, Sol. Phys., 59, 331

Lantos, P., \& Kundu, M. R. 1972, A\&A, 21, 119

Lindsey, C. A., \& Jefferies, J. T. 1991, ApJ, 383, 443

Lindsey, C., \& Kaminski, C. 1984, ApJ, 282, L103

Lindsey, C., \& Kopp, G. 1995, ApJ, 453, 517

Lindsey, C., \& Roellig, T. 1987, ApJ, 313, 877

Lindsey, C., Becklin, E. E., Jefferies, J. T., et al. 1984, ApJ, 281, 862

Lindsey, C. A., Yee, S., Roellig, T. L., et al. 1990, ApJ, 353, L53

Lindsey, C., Kopp, G., Clark, T. A., \& Watt, G. 1995, ApJ, 453, 511

Lites, B. W., Rutten, R. J., \& Kalkofen, W. 1993, ApJ, 414, 345

Loukitcheva, M., Solanki, S. K., Carlsson, M., \& Stein, R. F. 2004, A\&A, 419, 747

Loukitcheva, M., Solanki, S. K., \& White, S. M. 2006, A\&A, 456, 713

Lugten, J. B. 1995, BIMA Memo 38: Optical Design Parameters of BIMA's TIW Antennas

McAteer, R. T. J., Gallagher, P. T., Bloomfield, D. S., et al. 2004, ApJ, 602, 436

Plambeck, R. L. 2000, ALMA Memo Series, 321

Roellig, T. L., Becklin, E. E., Jefferies, J. T., et al. 1991, ApJ, 381, 288

Rutten, R. J., \& Uitenbroek, H. 1991, Sol. Phys., 134, 15

Shimabukuro, F. I., Wilson, W. J., Mori, T. T., \& Smith, P. L. 1975, Sol. Phys., 40,359

Simon, M., \& Shimabukuro, F. I. 1971, ApJ, 168, 525

Solanki, S. K. 2005, in Multi-Wavelength Investigations of Solar Activity, ed. A. V. Stepanov, E. E. Benevolenskaya, \& A. G. Kosovichev (Cambridge: Cambridge Univ. Press), IAU Symp., 223

Swanson, P. W., \& Hagen, J. P. 1975, Sol. Phys., 43, 57

Tolbert, C. W., Krause, L. C., \& Straiton, A. W. 1964, ApJ, 140, 306

Torrence, C., \& Compo, G. 1998, Bull. Am. Meteorol. Soc., 79, 61

Ulich, B. L., \& Haas, R. W. 1976, ApJS, 30, 247

Urpo, S., Hildebrandt, J., \& Krüger, A. 1987, Sol. Phys., 112, 119

Vernazza, J. E., Avrett, E. H., \& Loeser, R. 1976, ApJS, 30, 1

Vernazza, J. E., Avrett, E. H., \& Loeser, R. 1981, ApJS, 45, 635

Wedemeyer, S., Freytag, B., Steffen, M., Ludwig, H.-G., \& Holweger, H. 2004, A\&A, 414, 1121

Welch, W. J., Thornton, D. D., Plambeck, R. L., et al. 1996, Publ. Astron. Soc. Pacific, 108, 93

White, S. M., \& Kundu, M. R. 1994, in Infrared Solar Physics, ed. D. M. Rabin, J. T. Jefferies, \& C. Lindsey (Dordrecht: Kluwer Academic Publishers), IAU Symp., 154, 167

Zirin, H. 1996, Sol. Phys., 169, 313 